\title{
Generalized shape optimization of transient vibroacoustic problems using cut elements
}

\author{
Dilgen, Cetin Batur; Aage, Niels
}

Published in:

International Journal for Numerical Methods in Engineering

Link to article, DOI:

10.1002/nme.6591

Publication date:

2021

Document Version

Peer reviewed version

Link back to DTU Orbit

Citation (APA):

Dilgen, C. B., \& Aage, N. (2021). Generalized shape optimization of transient vibroacoustic problems using cut elements. International Journal for Numerical Methods in Engineering, 122, 1578-1601. https://doi.org/10.1002/nme.6591

\section{General rights}

Copyright and moral rights for the publications made accessible in the public portal are retained by the authors and/or other copyright owners and it is a condition of accessing publications that users recognise and abide by the legal requirements associated with these rights.

- Users may download and print one copy of any publication from the public portal for the purpose of private study or research.

- You may not further distribute the material or use it for any profit-making activity or commercial gain

- You may freely distribute the URL identifying the publication in the public portal

If you believe that this document breaches copyright please contact us providing details, and we will remove access to the work immediately and investigate your claim. 


\title{
Generalized shape optimization of transient vibroacoustic problems using cut elements
}

\author{
Cetin B. Dilgen I Niels Aage
}

Centre for Acoustic-Mechanical Micro Systems (CAMM), Department of

Mechanical Engineering, Technical

University of Denmark, Denmark

\section{Correspondence}

Cetin B. Dilgen, Technical University of

Denmark, Nils Koppels Allé, Building 404,

DK-2800, Denmark

Email: cedil@mek.dtu.dk
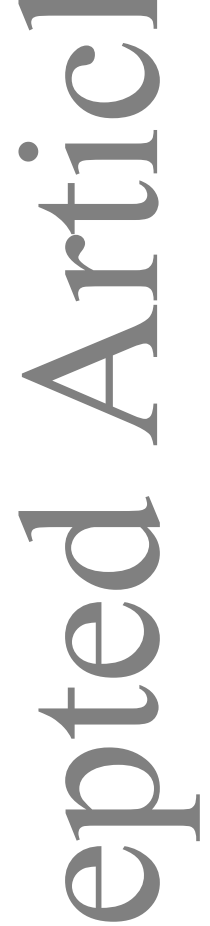

\begin{abstract}
This paper propose a generalized shape optimization method for transient coupled acoustic-mechanical interaction problems. The transient problem formulation allows to optimize for a broadband frequency content and the possibility to investigate transient phenomena such as pulse shaping. Throughout the work, the geometry is defined with the help of a nodal based design field, for which its zero level contour describes the interface between acoustic and structural domains. The approach utilizes a fixed background mesh to represent the geometry and a cut element immersed boundary method for the physical modelling. This modelling approach provides accurate solutions to the strongly coupled governing equations based on a special integration scheme. The optimization problem is solved using a gradient based optimizer and employs a fully discrete adjoint approach for calculating the sensitivity information. A numerical examination on the accuracy of the sensitivity analysis compares the fully discrete gradients to the commonly used semi-discrete adjoint approach for transient optimization revealing the importance of consistent sensitivities. The transient design formulation is validated on a $2 \mathrm{D}$ benchmark problem concerning the design of a time-harmonic acoustic partitioner. The developed framework is then applied for the design of vibroacoustic pulse shaping devices to demonstrate control of a transient phenomena.
\end{abstract}

KEYWORDS:

Vibroacoustics, Transients, Generalized shape optimization, Cut elements, Gradients, Level set methods

\section{1 | INTRODUCTION}

Shape and topology optimization ${ }^{1}$ have become widely popular in engineering design with ever widening application areas of in both academia and industry. One of the most popular approaches is density based topology optimization, which is a method to determine optimized material distributions that minimizes a given objective function. The key to the success of the density method is to relax the material representation by introducing intermediate densities, meaning that the material properties are commonly interpolated between solid and void phases. This approach readily facilitates the use of efficient gradient based optimizers such as the method of moving asymptotes (MMA) ${ }^{2}$. However, for certain problem types in which the interface plays a crucial role in the modelling, the physical interpretation of intermediate material realizations can pose severe and limiting issues $^{3}$. This can, for some problems, be mitigated through image processing filters ${ }^{4,5}$ and projection filters ${ }^{6,7}$ that serves to eliminate intermediate densities. These methods have paved the way for giga-scale structural optimization ${ }^{8}$ and made the density

This article has been accepted for publication and undergone fill peer review but has not been through the copyediting, typesetting, pagination and proofreading process which may lead to differences between this version and the Version of Record. Please cite this article as doi: $10.1002 /$ nme.6591 
approach a commonly used tool in industrial design. Although primarily used for structural optimization, the method has gained popularity in many other applications and different physics including fluid dynamics $\frac{9100}{\text {, acoustics } 1112 \mid 13114}$, transient wave

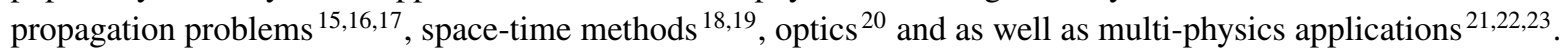

However, the presence of the intermediate densities in the optimized design (including those appearing during the optimization stage) can cause issues due to the lack of a clear boundary definition. Furthermore, for multi-physics problems coupled through an interface it is often crucial to have a smooth boundary definition due to an inherent sensitivity to sharp boundary variations, e.g. kinks. To this end, the so called staircase (pixelated) boundary representation introduces issues for density based topology optimization when it is used for coupled multi-physics problems ${ }^{3 / 24}$. Another issue for interface dependent design problems occurs after the optimization process. For example, for strongly coupled vibroacoustic problems, the final design's performance can be adversely affected after post-processing, especially for high frequency applications, due to a lack of physical interpretation of small regions of gray material or the smoothing of jagged edges. So although density based methods have been successfully applied to low frequency vibroacoustic design problems using a mixed finite element formulation ${ }^{25}$, the potential drawbacks as mentioned above advocates the use of design and modelling approaches capable of a clear and crisp interface representation.

An often used alternative to the density design methodology is the level set method 2627 . Such approaches implicitly defines the geometry from the zero contour of a level set function which in turn facilitates a crisp representation of the design with well-defined boundaries. However, it is important to remark that an accurate representation of the state fields at the interface is not given by the level set method itself, but is completely dependent on the underlying numerical model. Nonetheless, multiple Jariations of the level set approach has been used in the context of structural optimization for vibroacoustic problems including those that employ ersatz-type material models ${ }^{28}$, those that depends on extensive remeshing ${ }^{29}$ and those that use an advectiondiffusion equation for the design update ${ }^{30 \mid 31}$. For a detailed comparison of density, level set and evolutionary based topology optimization methods for vibroacoustic problems the reader is referred to the recent review paper ${ }^{32}$.

This work presents a level set based generalized shape optimization framework for transient vibroacoustic problems. The transient formulation of the vibroacoustic systems enables the optimization to be carried out for a broad frequency content while also allowing the possibility to investigate transient phenomena. The developed design method is based on the cut element topology optimization approach presented in ${ }^{[3]}$ which is extended to accommodate the coupled vibroacoustic state problem. The cut

element method can, to some extend, be seen as a special case of the finite cell method ${ }^{34}$ or X-FEM without enrichments $\sqrt{35 \sqrt[36]{36}}$ in which the entire background mesh in maintained at all times. To the best of the authors' knowledge, transient shape optimization of vibroacoustic problems using a cut element method combined with a fully discrete adjoint approach has not been demonstrated before. The thorough explanations of the level set parameterization, the utilized immersed boundary method and the discrete temporal adjoint method that is applicable to any time integration scheme are also among the contributions of the present work. The remainder of the paper is organized as follows: The governing equations with spatial and temporal discretizaIOU of the coupled physics are introduced in section 2 Section 3 describes the employed level set parameterization used for the optimization. The cut element method used for the accurate modelling of the coupling of structural and acoustic domains is explained in section 4 . Section 5 introduces the optimization problem and implementation aspects of the discrete adjoint method for the sensitivity analysis. In section 6 the discrete sensitivities are compared to the semi-discrete approach and the optimization setup for the develop framework is validated on a benchmark problem. The transient shape optimization for vibroacoustics problems are then demonstrated on acoustic pulse shaping examples, after which the findings are discussed and summarized.

\section{2 | GOVERNING EQUATIONS}

In this section, the governing equations that are used throughout the work considering the structural displacements and acoustic pressure are introduced. Since the cut element method is utilized for the modeling of the coupled system and the subsequent optimization, both the structural and acoustic fields are embedded in the domain $\Omega$ which consists of the entire computational domain as

$$
\Omega=\Omega_{s} \cup \Omega_{a}
$$

where $\Omega_{a}$ denotes the current acoustic region and $\Omega_{s}$ is the embedded structure. Similar to other fictitious domain approaches, such as density methods, both physics are solved in $\Omega$ which allows for maximum design freedom without the need for expensive re-meshing throughout the optimization process. The two physics are coupled through the interface $\Gamma_{a s}$ situated between $\Omega_{s}$ and $\Omega_{a}$. Hence the correct definition of the boundaries and the coupling conditions are important for the modeling accuracy. The specifics of the cut element approach is described in section 4. 
The governing equations for linear elasticity considering a time dependent motion without the presence of a body force can be written as

$$
\begin{array}{ll}
\rho_{s} \frac{\partial^{2} \mathbf{u}}{\partial t^{2}}-\nabla \cdot \boldsymbol{\sigma}+\rho_{s} \alpha_{d} \frac{\partial \mathbf{u}}{\partial t}-\nabla \cdot\left(\beta_{d} \frac{\partial \boldsymbol{\sigma}}{\partial t}\right)=0 & \text { in } \Omega \\
\mathbf{u}=0 & \text { in } \Gamma_{s d} \\
\mathbf{n}_{s} \cdot \boldsymbol{\sigma}=0 & \text { in } \Gamma_{s n} \\
\mathbf{n}_{s} \cdot \boldsymbol{\sigma}=p \mathbf{n}_{a} & \text { in } \Gamma_{a s}
\end{array}
$$

where $\rho_{s}$ is the density of the solid material, $\mathbf{u}$ is the displacement vector, $p$ is the acoustic pressure, $\mathbf{n}_{s}$ is the normal vector defined at the interface pointing outwards from the structural boundary, $\mathbf{n}_{a}$ is the normal vector pointing outwards from the acoustic domain into the structural region. In order to approximately reflect the loss mechanism of the real world, the Rayleigh damping is considered (the last two terms on the right hand side of the Eq. 2) in which $\alpha_{d}$ and $\beta_{d}$ are the Rayleigh damping parameters. Moreover, $\boldsymbol{\sigma}$ is the Cauchy stress vector which is defined as

$$
\boldsymbol{\sigma}=\boldsymbol{C}\left(E_{s}, v\right) \boldsymbol{\epsilon}
$$

Here, $\mathcal{C}\left(E_{s}, v\right)$ is the plane stress constitutive matrix, $E_{s}$ is the Young's modulus, $v$ is the Poisson's ratio and the strain vector $\boldsymbol{\epsilon}$ is defined as

$$
\boldsymbol{\epsilon}=\left[\frac{\partial u_{1}}{\partial x}, \frac{\partial u_{2}}{\partial y}, \frac{\partial u_{1}}{\partial y}+\frac{\partial u_{2}}{\partial x}\right]^{\mathrm{T}}
$$

For the structural domain, coupling from the acoustic domain is realized through the boundary condition written in Equation 5 which means that the acoustic pressure $p$ applies a pressure load onto the structural boundary.

The solution to the elasticity equation follows the cut element fictitious approach of 33 . That is, the solution of the displacements in the fictitious domain, which is the void phase in $\Omega_{a}$ for the structure, is obtained by altering the material properties of the considered solid as

$$
E_{s}=\alpha \tilde{E}_{s}, \quad \rho_{s}=\alpha \tilde{\rho}_{s}
$$

where $\alpha$ is a dimensionless, spatially varying contrast parameter and the tilde superscript is used to specify the original material properties of the solid. Throughout the work, $\alpha$ takes the values of unity for the structure domain and $10^{-8}$ for the void phase of the structure. We remark that the immersed boundary methods employed here shares many similarities to finite cell methods $\frac{\sqrt{37}}{37}$ and the density method for topology optimization 25 .

The acoustic pressure is governed by the Helmholtz equation

$$
\begin{array}{ll}
\frac{1}{K_{a}} \frac{\partial^{2} p}{\partial t^{2}}-\frac{1}{\rho_{a}} \nabla^{2} p=0 & \text { in } \quad \Omega \\
\mathbf{n}_{a} \cdot \nabla p=0 & \text { in } \Gamma_{a d} \\
\mathbf{n}_{a} \cdot \nabla p=\rho_{a} \frac{\partial^{2}\left(\mathbf{n}_{s} \cdot \mathbf{u}\right)}{\partial t^{2}} & \text { in } \quad \Gamma_{a s} \\
\mathbf{n}_{a} \cdot \nabla p+\frac{1}{c_{a}} \frac{\partial p}{\partial t}=\frac{2}{c_{a}} \frac{\partial p_{i n}}{\partial t} & \text { in } \quad \Gamma_{a r}
\end{array}
$$

Here $c_{a}$ is the speed of sound in the acoustic medium, $\rho_{a}$ is the density of the fluid, $K_{a}$ is the bulk modulus for the acoustic medium defined as $K_{a}=\rho_{a} c_{a}^{2}$. Hard-wall boundary condition is given in the Equation 10 and the coupling to the structural domain is given in Equation 11 which represents an acceleration boundary condition into the acoustic domain. Equation 12 describes an absorbing boundary condition with plane wave radiation where $p_{\text {in }}$ denotes the transient incoming pressure wave.

Similarly to the elasticity problem, the acoustic pressure solution in the fictitious domain, which is the rigid phase for the acoustic pressure defined in $\Omega_{s}$, is obtained by changing the material properties of the acoustic medium as

$$
K_{a}=\frac{\tilde{K}_{a}}{\alpha}, \quad \rho_{a}=\frac{\tilde{\rho}_{a}}{\alpha}
$$

Again, the contrast parameter $\alpha$ is set to unity for the acoustic domain and $10^{-8}$ for the rigid phase of the acoustic solution. Additional details on the accuracy and consequences of the use of contrast parameters in immersed boundary methods can be found in the following papers on elasticity ${ }^{1137}$, acoustics ${ }^{11}$ and vibroacoustics ${ }^{25}$, respectively. 


\section{1 | Spatial discretization}

The finite element discretization of the vibroacoustic system is obtained by a standard Galerkin procedure. However, note in the following that the continuous state variables have been replaced by $\overline{\mathbf{u}}$ and $\bar{p}$ such that the resulting discrete system can be written without overbars. The resulting weak form can be stated as the following integral expression

$$
\begin{gathered}
\rho_{s} \int_{\Omega} \delta \mathbf{u}^{\mathrm{T}} \ddot{\overline{\mathbf{u}}} \mathrm{d} \Omega+\int_{\Omega} \delta \boldsymbol{\epsilon}^{\mathrm{T}} \boldsymbol{C} \boldsymbol{\epsilon} \mathrm{d} \Omega+\rho_{s} \alpha_{d} \int_{\Omega} \delta \mathbf{u}^{\mathrm{T}} \dot{\overline{\mathbf{u}}} \mathrm{d} \Omega+\beta_{d} \int_{\Omega} \delta \boldsymbol{\epsilon}^{\mathrm{T}} \boldsymbol{C} \dot{\boldsymbol{\epsilon}} \mathrm{d} \Omega-\int_{\Gamma_{a s}} \delta \mathbf{u}^{\mathrm{T}} \bar{p} \mathbf{n}_{a} \mathrm{~d} \Gamma=0 \\
\frac{1}{K_{a}} \int_{\Omega} \delta p \ddot{\bar{p}} \mathrm{~d} \Omega+\frac{1}{\rho_{a}} \int_{\Omega}(\nabla \delta p)^{\mathrm{T}} \nabla \bar{p} \mathrm{~d} \Omega-\int_{\Gamma_{a s}} \delta p \mathbf{n}_{s}^{\mathrm{T}} \ddot{\overline{\mathbf{u}}} \mathrm{d} \Gamma+\frac{1}{\rho_{a} c_{a}} \int_{\Gamma_{a r}} \delta p \dot{\bar{p}} \mathrm{~d} \Gamma=\frac{2}{\rho_{a} c_{a}} \int_{\Gamma_{a r}} \delta p \frac{\partial p_{i n}}{\partial t} \mathrm{~d} \Gamma
\end{gathered}
$$

where the single and double dot notation denotes the first and the second time derivative of a variable, respectively. Furthermore, $\delta \boldsymbol{\epsilon}$ is the virtual strain, $\delta \mathbf{u}$ and $\delta p$ are the test functions for displacements and the pressure field. The computational domain is meshed with regular Q-4 elements and the continuous variables $\overline{\mathbf{u}}$ and $\bar{p}$ are approximated at the nodal points by their corresponding time independent linear shape functions $\mathbf{N}_{u}$ and $\mathbf{N}_{p}$, respectively.

$$
\begin{array}{lll}
\overline{\mathbf{u}}=\mathbf{N}_{u} \mathbf{u}, & \ddot{\overline{\mathbf{u}}}=\mathbf{N}_{u} \ddot{\mathbf{u}}, & \boldsymbol{\epsilon}=\mathbf{B}_{u} \mathbf{u} \\
\bar{p}=\mathbf{N}_{p} \mathbf{p}, & \overline{\bar{p}}=\mathbf{N}_{p} \dot{\mathbf{p}}, & \ddot{\bar{p}}=\mathbf{N}_{p} \ddot{\mathbf{p}}
\end{array}
$$

where $\mathbf{B}_{u}$ is the linear strain-displacement matrix. Inserting the above definitions into the weak forms (Eqs. 14 and 15 ) and employing the standard Galerkin procedure ${ }^{\frac{38}{38}}$ where the test functions are replaced by the shape functions of the variables, the discrete weak form of the coupled system can be written as

$$
\underbrace{\left[\begin{array}{cc}
\mathbf{M}_{\mathbf{s}} & 0 \\
\mathbf{S}^{\mathrm{T}} & \mathbf{M}_{\mathbf{a}}
\end{array}\right]}_{\mathbf{M}}\left[\begin{array}{c}
\ddot{\mathbf{u}} \\
\ddot{\mathbf{p}}
\end{array}\right]+\underbrace{\left[\begin{array}{ccc}
\mathbf{C}_{\alpha}+\mathbf{C}_{\beta} & 0 \\
0 & \mathbf{C}_{r}
\end{array}\right]}_{\mathbf{C}}\left[\begin{array}{c}
\dot{\mathbf{u}} \\
\dot{\mathbf{p}}
\end{array}\right]+\underbrace{\left[\begin{array}{cc}
\mathbf{K}_{\mathbf{s}} & -\mathbf{S} \\
0 & \mathbf{K}_{\mathbf{a}}
\end{array}\right]}_{\mathbf{K}}\left[\begin{array}{l}
\mathbf{u} \\
\mathbf{p}
\end{array}\right]=\left[\begin{array}{l}
0 \\
\mathbf{g}
\end{array}\right]
$$

where the discretized finite element matrices are identified as

$$
\begin{aligned}
& \mathbf{K}_{s}=\int_{\Omega} \mathbf{B}_{u}^{T} C \mathbf{B}_{u} \mathrm{~d} \Omega, \quad \mathbf{M}_{s}=\rho_{s} \int_{\Omega} \mathbf{N}_{u}^{T} \mathbf{N}_{u} \mathrm{~d} \Omega, \quad \mathbf{S}=\int_{\Gamma_{a s}} \mathbf{N}_{u}^{T} \mathbf{n}_{a} \mathbf{N}_{p} \mathrm{~d} \Gamma_{a s} \\
& \mathbf{C}_{\alpha}=\rho_{s} \alpha_{d} \int_{\Omega} \mathbf{N}_{u}^{T} \mathbf{N}_{u} \mathrm{~d} \Omega, \quad \mathbf{C}_{\beta}=\beta_{d} \int_{\Omega} \mathbf{B}_{u}^{T} \boldsymbol{C} \mathbf{B}_{u} \mathrm{~d} \Omega \\
& \mathbf{K}_{a}=\frac{1}{\rho_{a}} \int_{\Omega}\left(\nabla \mathbf{N}_{p}\right)^{T} \nabla \mathbf{N}_{p} \mathrm{~d} \Omega, \quad \mathbf{M}_{a}=\frac{1}{K_{a}} \int_{\Omega} \mathbf{N}_{p}^{T} \mathbf{N}_{p} \mathrm{~d} \Omega \\
& \mathbf{C}_{r}=\frac{1}{c_{a} \rho_{a}} \int_{\Gamma_{a r}} \mathbf{N}_{p}^{T} \mathbf{N}_{p} \mathrm{~d} \Gamma_{a s}, \quad \mathbf{g}=\frac{2}{c_{a} \rho_{a}} \int_{\Gamma_{a r}} \mathbf{N}_{p}^{T} \frac{\partial p_{i n}}{\partial t} \mathrm{~d} \Gamma_{a n}
\end{aligned}
$$

We remark that the cut element approach used in this work requires a slightly modified version of the standard numerical quadrature. This will be presented in section 4.1

\section{2 | Time integration}

After the spacial discretization the coupled system has the following semi discrete form

$$
\mathbf{M} \ddot{\mathbf{v}}^{n}+\mathbf{C} \dot{\mathbf{v}}^{n}+\mathbf{K} \mathbf{v}^{n}=\mathbf{h}^{n}
$$

where the contents of the combined mass matrix $\mathbf{M}$, damping matrix $\mathbf{C}$ and stiffness matrix $\mathbf{K}$ are given in Equation 18 . Here, the superscript $n$ denotes current time, the vector $\mathbf{v}$ is the solution vector $\mathbf{v}=[\mathbf{u}, \mathbf{p}]^{T}$ and the vector $\mathbf{h}=[0, \mathbf{g}]^{T}$ is the load vector for the given dynamic system. Throughout the presented work a Newmark algorithm ${ }^{39}$, i.e. an implicit time-stepping scheme, 
is used for the temporal discretization. Here the first and the second time derivatives of the solution vector $\mathbf{v}$ are expressed as

$$
\begin{aligned}
\dot{\mathbf{v}}^{n} & =a_{1} \dot{\mathbf{v}}^{n-1}+a_{2} \ddot{\mathbf{v}}^{n-1}+a_{3}\left(\mathbf{v}^{n}-\mathbf{v}^{n-1}\right) \\
\ddot{\mathbf{v}}^{n} & =-a_{4} \dot{\mathbf{v}}^{n-1}-a_{5} \ddot{\mathbf{v}}^{n-1}+a_{6}\left(\mathbf{v}^{n}-\mathbf{v}^{n-1}\right)
\end{aligned}
$$

where the parameters $a_{1}$ to $a_{6}$ are the Newmark parameters. Substituting the above definitions (Eqs. 24 and 25) into the semi discrete coupled system given in Equation 23, leads to the following fully discrete linear system of equations to be solved for $\mathbf{v}^{n}$ for the current time $n$

$$
\hat{\mathbf{K}} \mathbf{v}^{n}=\hat{\mathbf{h}}^{n}
$$

where $\hat{\mathbf{K}}$ and $\hat{\mathbf{h}}^{n}$ are the so called effective stiffness matrix and load vector, respectively. $\hat{\mathbf{K}}$ and $\hat{\mathbf{h}}^{n}$ are defined as

$$
\begin{aligned}
& \hat{\mathbf{K}}=\mathbf{K}+a_{6} \mathbf{M}+a_{3} \mathbf{C} \\
& \hat{\mathbf{h}}^{n}=\mathbf{h}^{n}+\mathbf{M}\left(a_{4} \dot{\mathbf{v}}^{n-1}+a_{5} \ddot{\mathbf{v}}^{n-1}+a_{6} \mathbf{v}^{n-1}\right)+\mathbf{C}\left(-a_{1} \dot{\mathbf{v}}^{n-1}-a_{2} \ddot{\mathbf{v}}^{n-1}+a_{3} \mathbf{v}^{n-1}\right)
\end{aligned}
$$

Moreover, the Newmark parameters are given as

$$
\begin{array}{ll}
a_{1}=1-\frac{\tilde{\gamma}}{\tilde{\beta}}, & a_{2}=\left(1-\frac{\tilde{\gamma}}{2 \tilde{\beta}}\right) \Delta t, \quad a_{3}=\frac{\tilde{\gamma}}{\tilde{\beta} \Delta t} \\
a_{4}=\frac{1}{\tilde{\beta} \Delta t}, & a_{5}=\frac{1}{2 \tilde{\beta}}-1, \quad a_{6}=\frac{1}{\tilde{\beta} \Delta t^{2}}
\end{array}
$$

where $\Delta t$ is the time step size. Following $\frac{40}{\beta}, \tilde{\beta}$ and $\tilde{\gamma}$ are chosen such that the utilized time-stepping scheme is unconditionally stable which are given as

$$
\tilde{\beta}=\frac{1}{4}, \quad \tilde{\gamma}=\frac{1}{2}
$$

For all presented examples in this paper the initial conditions $\mathbf{v}^{0}$ and $\dot{\mathbf{v}}^{0}$ are set to zero, however, this does not need to be the case. Therefore, to avoid the loss of generality, the initial condition for the second time derivative of the solution vector $\ddot{\mathbf{v}}^{0}$ is found by solving

$$
\mathbf{M} \ddot{\mathbf{v}}^{0}=\mathbf{h}^{0}-\mathbf{C \dot { v }}^{0}-\mathbf{K} \mathbf{v}^{0}
$$

Finally, we remark that the presented temporal discretization is not limited to the Newmark algorithm and that proper choices for $a_{i}$ could turn the scheme into e.g. an implicit Euler method. The numerical model is implemented in $\mathrm{C}^{++}$where the PETSc 1:1- $\operatorname{ary}{ }^{4142[43}$ is utilized for its parallel data arrangement. The solution of equation 26 is obtained using the parallel direct solver MUMPS 4445 .

\section{3 | DESIGN PARAMETERIZATION}

The design parameterization used in the presented work follows the level set approach based on density methods presented in ${ }^{33}$. That is, a continuous mathematical nodal design field $s$ is introduced similar to that of classical density methods, i.e.

$$
0 \leq s \leq 1
$$

This choice of bounds for the design field means that standard optimizers such as the Methods of Moving Asymptotes can readily be applied ${ }^{2}$. However, to ensure that the interface does not progress to fast during the optimization process, a mesh dependent mapping is used to introduce the element size to the parameterization as

$$
-0.5 h_{e} \leq \tilde{s}(s) \leq 0.5 h_{e}
$$

where $h_{e}$ is the average element side length. The last step before feeding the design field to the finite element analysis is to regularize the problem with a convolution filter. This is included to regularize the problem by increasing the zone of influence of the design variables, and hence also the sensitivities, which stabilizes and speeds up the design process. The filter, which is applied on $\widetilde{s}(s)$, is realized using a Helmholtz type differential equation $\frac{46}{6}$, which reads

$$
-r^{2} \nabla^{2} \bar{s}_{c}+\bar{s}_{c}=\tilde{s}_{c} \text { in } \Omega
$$


where the subscript $c$ specifies variables that are defined in cell centers and $r$ is the filtering radius. Here it is noted that, for stability, the filter equation is solved using a finite volume method in which the variables are stored in cell centers and represented as piece-wise constant. That is, if the filter equations was solved using a finite element scheme, oscillations will occur in its solution for small filter radii. This shortcoming is completely alleviated with the finite volume scheme. However, the finite volume scheme comes at a cost, which is that the field $\tilde{s}(s)$ is first interpolated from nodes to cell centers. After filtering, the cell centered field $\bar{s}_{c}$ is interpolated back to the nodes leading to the physical design field $\bar{s}$. It is noted that the two interpolation operations (from nodes to the element centers and from element centers to the nodes) provide extra implicit filtering that is always active even when the filtering radius $r$ is set to zero.

The physical design field, $\bar{s}$, can now be used as a level set field to determine if a given part of $\Omega$ is either acoustic, structural or the interface based on the following rule

$$
\begin{array}{ll}
\bar{s}(x)>0, & x \in \Omega_{s} \quad \text { (structural domain) } \\
\bar{s}(x)=0, & x \in \Gamma_{a s} \text { (interface) } \\
\bar{s}(x)<0, & x \in \Omega_{a} \text { (acoustic domain) }
\end{array}
$$

An illustrative example of the function $\bar{s}$ can be seen in Figure 1 where the embedded structural and acoustic domains are identified along with the shared interface between the two domains. Figure 1 also shows the projection of $\bar{s}$ onto a fixed background finite element mesh.

(a)

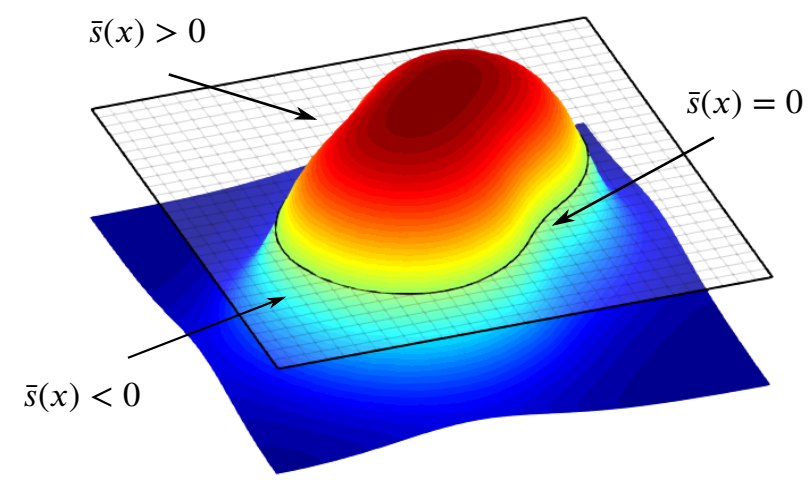

(b)

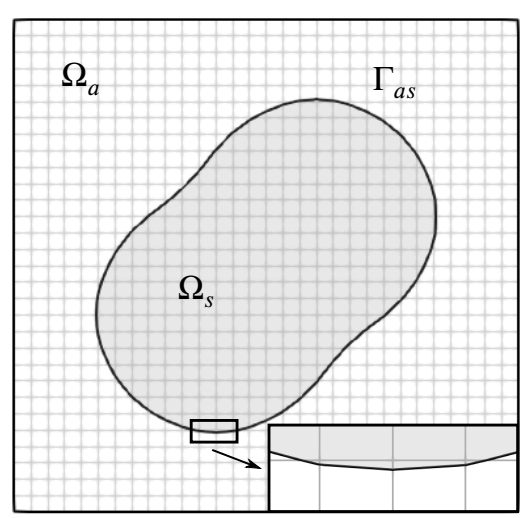

FIGURE 1 An example level set function showing the embedded physical domains. (a) shows the rule that is used for specifying different physical domains embedded with the level set function, (b) shows the extracted structure from the zero iso-curve of the level set function on a fixed finite element mesh.

As shown in Figure $1 \mathrm{~b}$, since both $s$ and $\bar{s}$ use linear shape functions, the resulted non-conforming boundary is represented by linear straight curves inside cut elements. We note that a 2D level set method cannot introduce holes without a auxiliary hole generation scheme, e.g. topological derivatives, but that a 3D version of the presented approach could. Therefore the proposed $2 \mathrm{D}$ method is termed a generalized shape optimization method. Finally, we remark that the proposed design parameterization does not introduce a minimum length scale to the design problem. Although this could be included following the robust approach e.g. ${ }^{733}$, this is deemed outside the scope of this manuscript since the focus is on the numerical methodology allowing for transient vibroacoustic generalized shape optimization optimization.

\section{4 | CUT ELEMENT INTEGRATION}

Having introduced the design parameterization, this section describes the modeling of non-conforming boundaries through a simple cut element method. That is, instead of re-meshing with conforming elements along the interface, which is expensive and difficult to parallelize, the cut element method realizes the exact boundary representation (piecewise linear) by special integration of the elements cut by the zero contour of the level set field. The utilized method does not enrich the shape functions 
or add additional degrees of freedom to the system. Hence, it is easily parallelizable as the integration that is done in the cut elements are only local operations.

The cut element procedure can be summarized as follows. Firstly the cut elements are identified by looping through all elements and checking for a sign change in the nodal level set field. Then, a triangulation algorithm is employed to sub-divide the intersected element into triangles with respecting the partial interface inside the element. The present work utilizes the marching squares algorithm ${ }^{47}$ in which the nodal physical level set values are used to determine the location of the zero level contour (intersecting boundary) along the edges of the element. The subsequent triangulation is done from a pre-generated look-up table since in marching squares algorithm there are only 16 unique cases a Quad element can be intersected. Figure 2 illustrates the cut element identification process.
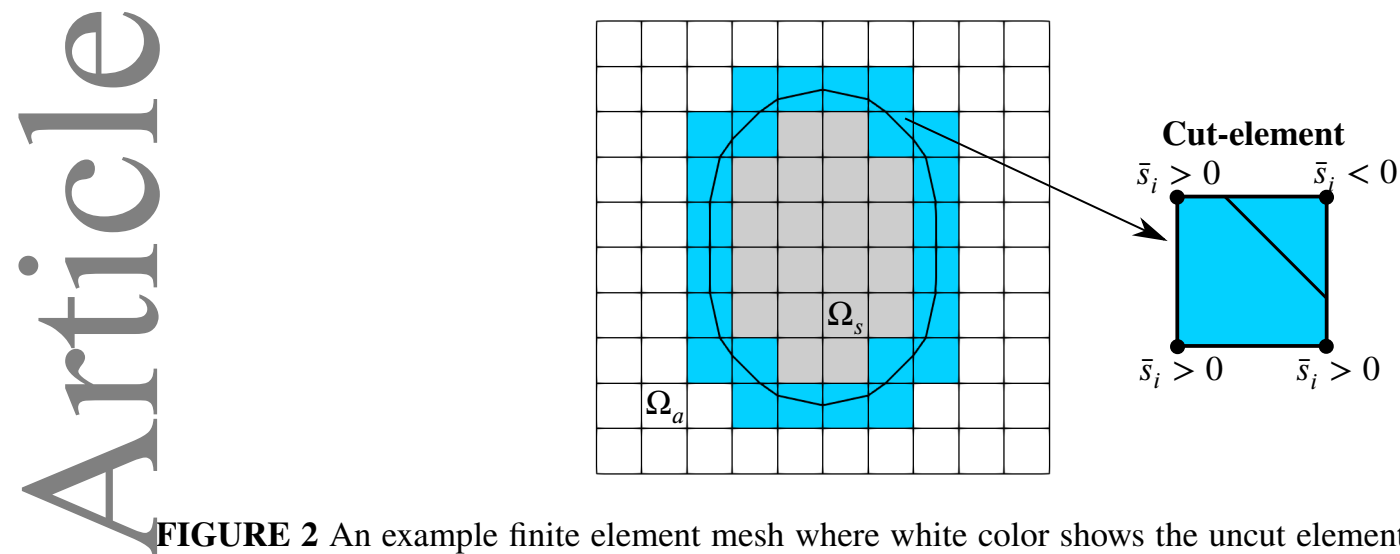

FIGURE 2 An example finite element mesh where white color shows the uncut elements in the acoustic domain, gray color shows the uncut elements in the solid region and the blue color shows the cut elements. Figure also shows an example of identifying a cut element from its nodal level set values.

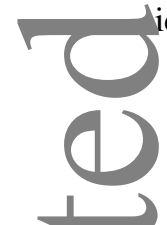

\section{1 | Sub-integration}

Th/s section describes the sub-element integration which is carried out for the elements that are cut by a non-conforming boundary. Cut elements utilize the Gaussian quadrature rule for capturing the effect of the boundary description through weighted integration. As such, it is important to correctly place the integration points in the sub-elements of the parent cut element. Here it is noted that the triangulation in the cut elements are only carried out in order to correctly place the integration points in the sub-elements and the interface line inside the parent cut element.

Figure $3 \mathrm{a}$ shows an example of a cut element where the sub-elements are obtained through triangulation. Figures $3 \mathrm{~b}$ to $3 \mathrm{c}$ illustrates mapping the Gauss points defined at the reference domain of the sub-triangle element to the reference domain of the parent element where the integration is carried out. The reader is referred to $\frac{33}{3}$ for details regarding the mapping of integration points. Following the nomenclature of Figure 3 the element matrix contribution for the stiffness part of linear system becomes

$$
\mathbf{K}_{s}=\sum_{i=1}^{n g} \mathbf{B}_{u}^{T}\left(\xi_{Q, i}, \eta_{Q, i}\right) \mathcal{C}\left(E_{s}, v\right) \mathbf{B}_{u}\left(\xi_{Q, i}, \eta_{Q, i}\right) W_{Q, i}\left\|J\left(\xi_{Q, i}, \eta_{Q, i}\right)\right\|
$$

Where the weight $W_{Q, i}$ is modified to take into account the area scaling between sub-triangle and parent element. Since the vibroacoustic system also needs to include the coupling at the material interface, a similar procedure is applied to the line integrals. The process is illustrated in Figure 4 and the subsequent integration over the quadrilateral is performed as

$$
\mathbf{S}=\sum_{i=1}^{n g} \mathbf{N}_{u}^{T}\left(\xi_{Q, i}, \eta_{Q, i}\right) \mathbf{n}_{a} \mathbf{N}_{p}\left(\xi_{Q, i}, \eta_{Q, i}\right) W_{Q, i} \frac{l}{2}
$$

where $l$ is the length of the coupling boundary. Again, it should be emphasized that the contribution from the integration goes to the original degrees of freedoms of the parent element. 


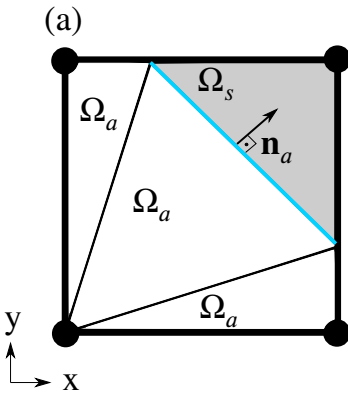

(b)

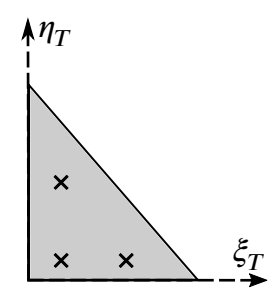

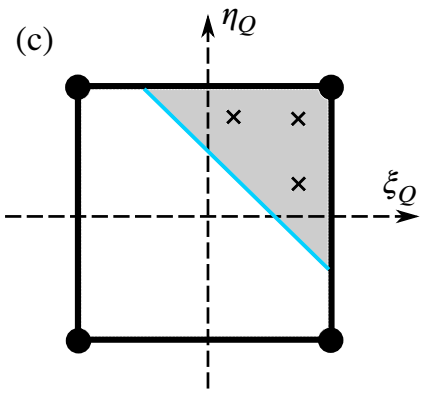

FIGURE 3 Illustration of a sub-cell integration in a cut element. (a) physical domains are identified for the triangulated cut elements where white color is the acoustic domain, gray color is the structural domain and the blue line is the interface. (b) Gauss points are inserted in an iso-parametric triangle sub-element in local coordinates. (c) Gauss points of the iso-parametric triangle sub-element are mapped to the reference domain of the parent element for integration.

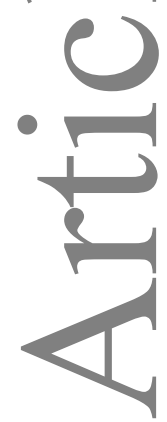

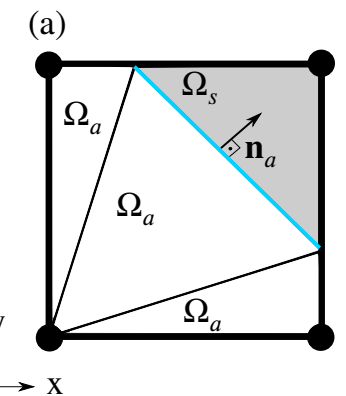

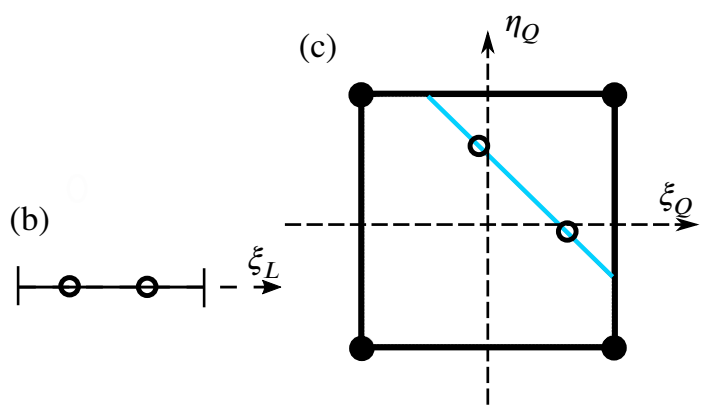

FIGURE 4 Illustration of a sub-cell interface integration in a cut element. (a) physical domains are identified for the triangulated cut elements where white color is the acoustic domain, gray color is the structural domain and the blue line is the interface. (b) Gauss points are inserted in an iso-parametric line sub-element in its local coordinates. (c) Gauss points of the iso-parametric line sub-element are mapped to the reference domain of the parent element for integration.

\section{2 | Modelling validation}

For completion, this section provides a validation study in which the implemented cut element method is compared to a body-fitted (segregated) finite element method obtained using COMSOL Multiphysics ${ }^{48}$. In order to carry out the COMSOL calculation, the structure is extracted from the cut element representation using the zero iso-level, which is then imported into the COMSOL software where a body-fitted mesh with linear triangle elements is constructed.

The test problem can be seen in Figure 5a which shows a partitioning structure containing two holes with different sizes and shapes. The example problem is constructed to ensure that most of the possible cut configurations are encountered, since these are likely to happen when employing the proposed shape optimization procedure. The specifics of the acoustic domain is set to air with a speed of sound and a density of $c_{a}=343 \mathrm{~m} / \mathrm{s}$ and $\rho_{a}=1.21 \mathrm{~kg} / \mathrm{m}^{3}$, respectively. The material properties of the solid domain are: $E_{s}=10^{7} \mathrm{~Pa}, \rho_{s}=2000 \mathrm{~kg} / \mathrm{m}^{3}$ and $v=0.4$. The system is excited at the inlet of the channel (shown as a blue line in Figure 5a with a Dirichlet boundary condition realizing an incoming acoustic sinusoidal plane wave with an amplitude of $p_{\text {in }}=1 \mathrm{~Pa}$ at $1900 \mathrm{~Hz}$. The outlet of the channel (shown as a red line in Figure 5a) is treated as an open boundary using an absorbing boundary condition. Both the top and the bottom boundaries are set as hard-wall condition for the acoustic pressure and the structure is considered to be clamped. The calculations are run for a total of $0.012 \mathrm{~s}$ using a step size of $\Delta t=2 \times 10^{-5} \mathrm{~s}$.

The result of the study can be seen in Figure $5 \mathrm{~b}$ which shows the averaged transmitted acoustic pressure at the channel outlet plotted as a function of time. From this figure it is qualitatively observed that the response of the coupled system obtained from the immersed boundary cut element method closely follows the segregated analysis done with a body fitted mesh in COMSOL. Notable differences between the two responses occur at the peaks of the transmitted acoustic pressure. These differences can be attributed to the usage of different meshes and to the fact that the extracted geometry is not completely preserved during the body-fitted re-meshing procedure used by COMSOL. It is also worth noting that the developed transient framework utilizes 


\section{5 | OPTIMIZATION PROBLEM}

In this section a generic optimization problem for a transient optimization is given where an objective function is sought to be minimized while satisfying the considered constraints. The optimization problem is written as

$$
\begin{aligned}
& \min _{\mathbf{s}} \Phi\left(\overline{\mathbf{s}}, \mathbf{U}^{0}(\overline{\mathbf{s}}), \ldots, \mathbf{U}^{N}(\overline{\mathbf{s}})\right)=\sum_{n=0}^{N} \phi^{n}\left(\overline{\mathbf{s}}, \mathbf{U}^{n}(\overline{\mathbf{s}})\right) \\
& \text { s.t. } \quad \mathbf{R}^{n}\left(\overline{\mathbf{s}}, \mathbf{U}^{n}(\overline{\mathbf{s}})\right)=0, \quad \text { for } n=0,1, \ldots, N \\
& \psi_{i}\left(\overline{\mathbf{s}}, \mathbf{U}^{0}(\overline{\mathbf{s}}), \ldots, \mathbf{U}^{N}(\overline{\mathbf{s}})\right) \leq 0, \quad \text { for } \quad i=0,1, \ldots, m \\
& s_{\min } \leq \mathbf{s} \leq s_{\max }
\end{aligned}
$$

(1)

where $\mathbf{s}$ is the vector of design variables, $\Phi$ is the objective function, $\mathbf{U}^{n}$ is the vector of state variables, the residual vector $\mathbf{R}^{n}$ is obtained from the discretization of the governing equations (sections 2.1 and 2.2, $N$ denotes the total number of time steps considered in the optimization, $\psi_{i}$ are the $m$ possible inequality constraints, $s_{\min }=0$ and $s_{\max }=1$ are the bounds for the design variables.

As a consequence of using the Newmark algorithm for temporal discretization, the vector $\mathbf{U}^{n}$ contains the state variables and their first and the second time derivatives. Likewise, the residual vector $\mathbf{R}^{n}$ contains the corresponding residuals which are written as

$$
\mathbf{R}^{n}=\left[\begin{array}{c}
\mathbf{r}_{1}^{n} \\
\mathbf{r}_{2}^{n} \\
\mathbf{r}_{3}^{n}
\end{array}\right], \quad \mathbf{U}^{n}=\left[\begin{array}{c}
\mathbf{v}^{n} \\
\dot{\mathbf{v}}^{n} \\
\ddot{\mathbf{v}}^{n}
\end{array}\right]
$$

where the individual residual vectors can be identified from the temporal discretization using the equations 24 to 28 as

$$
\begin{aligned}
& \mathbf{r}_{1}^{n}=\left[\mathbf{K}+a_{6} \mathbf{M}+a_{3} \mathbf{C}\right] \mathbf{v}^{n}-\left[a_{6} \mathbf{M}+a_{3} \mathbf{C}\right] \mathbf{v}^{n-1}-\left[a_{4} \mathbf{M}-a_{1} \mathbf{C}\right] \dot{\mathbf{v}}^{n-1}+\left[a_{2} \mathbf{C}-a_{5} \mathbf{M}\right] \ddot{\mathbf{v}}^{n-1}-\mathbf{h}^{n} \\
& \mathbf{r}_{2}^{n}=\dot{\mathbf{v}}^{n}-a_{1} \dot{\mathbf{v}}^{n-1}-a_{2} \ddot{\mathbf{v}}^{n-1}-a_{3}\left[\mathbf{v}^{n}-\mathbf{v}^{n-1}\right] \\
& \mathbf{r}_{3}^{n}=\ddot{\mathbf{v}}^{n}+a_{4} \dot{\mathbf{v}}^{n-1}+a_{5} \ddot{\mathbf{v}}^{n-1}-a_{6}\left[\mathbf{v}^{n}-\mathbf{v}^{n-1}\right]
\end{aligned}
$$

The optimization problem (Eqs. 39 to 42 ) is solved using the Method of Moving Asymptotes (MMA) ${ }^{2}$. The present works uses a parallel PETSc implementation of the optimizer, c.f. $\stackrel{4950 .}{.}$

\section{1 | Sensitivity analysis}

The sensitivity analysis needed for the application of a gradient based optimizer is here presented considering the generic objective function stated in Eq. 39. To this end a fully discrete sensitivity analysis is employed rather than the commonly used

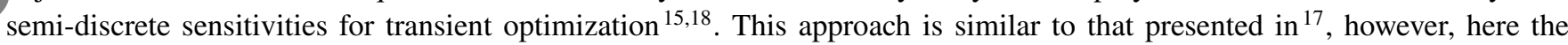
derivation is written in a general matrix form to ease the implementation and extension by others. The result is valid for any objective function and the use of a fully discrete sensitivity analysis ensures that the sensitivities always are consistent. Moreover, the quality of the calculated gradients does not depend on the utilized time step size or the time integration scheme since there is no time integration for the adjoint equation. The process of obtaining the sensitivity expression follows the standard engineering approach. That is, first the objective function is augmented using a vector of Lagrange multiplier $\boldsymbol{\Lambda}$ and the resulted Lagrangian function $\mathcal{L}$ is written as

$$
\mathcal{L}=\sum_{n=0}^{N} \phi^{n}\left(\overline{\mathbf{s}}, \mathbf{U}^{n}(\overline{\mathbf{s}})\right)+\boldsymbol{\Lambda}^{n} \mathbf{R}^{n}\left(\overline{\mathbf{s}}, \mathbf{U}^{n}(\overline{\mathbf{s}})\right)
$$

Here it is used that the residual is always satisfied, i.e. $\mathbf{R}^{n}=0$, for any given time step $n$. As with the state variable $\mathbf{U}^{n}$, the Lagrange multiplier vector consists of three fields as

$$
\Lambda^{n}=\left[\begin{array}{c}
\lambda^{n} \\
\dot{\lambda}^{n} \\
\ddot{\lambda}^{n}
\end{array}\right]
$$


The derivative of the Lagrangian function with respect to the physical level set field $\overline{\mathbf{s}}$ can be written as

$$
\begin{aligned}
\frac{\mathrm{d} \mathcal{L}}{\mathrm{d} \overline{\mathbf{s}}} & =\sum_{n=0}^{N} \frac{\partial \phi^{n}}{\partial \overline{\mathbf{s}}}+\frac{\partial \phi^{n}}{\partial \mathbf{U}^{n}} \frac{\partial \mathbf{U}^{n}}{\partial \overline{\mathbf{s}}}+\Lambda^{n T}\left[\frac{\partial \mathbf{R}^{n}}{\partial \overline{\mathbf{s}}}+\frac{\partial \mathbf{R}^{n}}{\partial \mathbf{U}^{n}} \frac{\partial \mathbf{U}^{n}}{\partial \overline{\mathbf{s}}}\right] \\
& =\sum_{n=0}^{N} \frac{\partial \phi^{n}}{\partial \overline{\mathbf{s}}}+\Lambda^{n T} \frac{\partial \mathbf{R}^{n}}{\partial \overline{\mathbf{s}}}+\underbrace{\left[\frac{\partial \phi^{n}}{\partial \mathbf{U}^{n}}+\Lambda^{n T} \frac{\partial \mathbf{R}^{n}}{\partial \mathbf{U}^{n}}\right]}_{=0} \frac{\partial \mathbf{U}^{n}}{\partial \overline{\mathbf{s}}}
\end{aligned}
$$

Since the Lagrangian vector can be freely chosen the calculation of the partial derivative $\frac{\partial \mathbf{U}^{n}}{\partial \overline{\mathbf{s}}}$ can be avoided if the underlined part of the above equation 50 is set to zero. This gives rise to the following set of adjoint equations for each time step

$$
\begin{gathered}
\left(\frac{\partial \mathbf{R}^{n}}{\partial \mathbf{U}^{n}}\right)^{T} \Lambda^{n}=-\frac{\partial \phi^{n}}{\partial \mathbf{U}^{n}}, \quad \text { for } n=0,1, \ldots, N \\
\frac{\partial \mathbf{R}^{T}}{\partial \mathbf{U}} \boldsymbol{\Lambda}=-\frac{\partial \phi}{\partial \mathbf{U}}
\end{gathered}
$$

- $\quad$ where the superscript $n$ is dropped to indicate that Eq. 52 now contains the all time steps used in the optimization. The partial derivative of the residual vector with respect to the state variables $\frac{\partial \mathbf{R}}{\partial \mathbf{U}}$ then has the following form

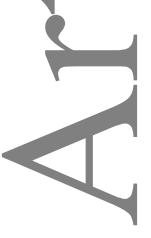

$$
\frac{\partial \mathbf{R}}{\partial \mathbf{U}}=\left[\begin{array}{cccccc}
\mathbf{A}_{0}^{\mathbf{U}^{0}} & & & & \\
\mathbf{B}_{1}^{\mathbf{U}^{0}} & \mathbf{A}_{1}^{\mathbf{U}^{1}} & & & \\
& \ddots & \ddots & & \\
& & \ddots & \ddots & & \\
& & & \mathbf{B}_{N-1}^{\mathbf{U}^{\mathrm{N}-2}} & \mathbf{A}_{N}^{\mathbf{U}^{\mathrm{N}-1}} & \\
& & & & \mathbf{B}_{N}^{\mathbf{U}^{\mathrm{N}-1}} & \mathbf{A}_{N}^{\mathbf{U}^{\mathrm{N}}}
\end{array}\right]
$$

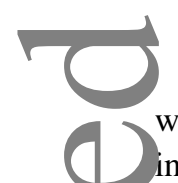

where the subscripts denote the current time step and the superscripts show the corresponding state variables for the sub-matrices in $\frac{\partial \mathbf{R}}{\partial \mathbf{U}}$. Here, the sub-matrices $\mathbf{A}$ and $\mathbf{B}$ are written in general form and will vary depending on the employed time integration scheme. Considering the Newmark algorithm, the $\mathbf{A}$ and $\mathbf{B}$ sub-matrices are identified from the residuals written in equations 44 to 46 For a time step greater than zero, i.e. $n>0$, these are constant and can be written as

$$
\mathbf{A}=\left[\begin{array}{cc}
{\left[\mathbf{K}+a_{6} \mathbf{M}+a_{3} \mathbf{C}\right]} & \\
-a_{3} \mathbf{I} & \mathbf{I} \\
-a_{6} \mathbf{I} & \mathbf{I}
\end{array}\right], \quad \mathbf{B}=\left[\begin{array}{ccc}
{\left[-a_{6} \mathbf{M}-a_{3} \mathbf{C}\right]} & {\left[-a_{4} \mathbf{M}+a_{1} \mathbf{C}\right]} & {\left[-a_{5} \mathbf{M}+a_{2} \mathbf{C}\right]} \\
a_{3} \mathbf{I} & -a_{1} \mathbf{I} & -a_{2} \mathbf{I} \\
a_{6} \mathbf{I} & a_{4} \mathbf{I} & a_{5} \mathbf{I}
\end{array}\right]
$$

Since these are constant, they can be assembled once and reused throughout the optimization cycle. Considering arbitrary initial conditions of the state equations, i.e. non-zero $\mathbf{v}^{0}$ and $\dot{\mathbf{v}}^{0}$, the initial condition of $\ddot{\mathbf{v}}^{0}$ is found by solving the equation 32 . This can be stated using the matrix $\mathbf{A}_{0}$, i.e.

$$
\mathbf{A}_{0}=\left[\begin{array}{lll}
\mathbf{I} & & \\
& \mathbf{I} & \\
\mathbf{K} & \mathbf{C} & \mathbf{M}
\end{array}\right]
$$

As a consequence of the transpose operation on $\frac{\partial \mathbf{R}}{\partial \mathbf{U}}$ in the adjoint equation (Eq. 52, the adjoint equation is decomposed into reverse pseudo time steps as

$$
\begin{aligned}
(\mathbf{A})^{T} \boldsymbol{\Lambda}^{N}= & -\frac{\partial \phi^{N}}{\partial \mathbf{U}} \\
(\mathbf{A})^{T} \boldsymbol{\Lambda}^{N-1}= & -\frac{\partial \phi^{N-1}}{\partial \mathbf{U}}-(\mathbf{B})^{T} \boldsymbol{\Lambda}^{N} \\
& \vdots \\
\left(\mathbf{A}_{0}\right)^{T} \boldsymbol{\Lambda}^{0}= & -\frac{\partial \phi^{0}}{\partial \mathbf{U}}-(\mathbf{B})^{T} \boldsymbol{\Lambda}^{1}
\end{aligned}
$$


This is the same type of reversal in time that is seen for the usual semi-discrete temporal adjoint approach. Now, having found the Lagrange multiplier $\boldsymbol{\Lambda}$ which satisfies the adjoint equation (Eq. 52), the final sensitivity of the objective function is calculated as follows

$$
\frac{\mathrm{d} \Phi}{\mathrm{d} \overline{\mathbf{s}}}=\frac{\partial \phi^{0}}{\partial \overline{\mathbf{s}}}+\boldsymbol{\Lambda}^{0^{T}}\left[\frac{\partial \mathbf{A}_{\mathbf{0}}}{\partial \overline{\mathbf{s}}} \mathbf{U}^{0}\right]+\sum_{n=1}^{N} \frac{\partial \phi^{n}}{\partial \overline{\mathbf{s}}}+\boldsymbol{\Lambda}^{n T}\left[\frac{\partial \mathbf{A}}{\partial \overline{\mathbf{s}}} \mathbf{U}^{n}+\frac{\partial \mathbf{B}}{\partial \overline{\mathbf{s}}} \mathbf{U}^{n-1}\right]
$$

As it can be seen from the final sensitivity expression, the state variables calculated from the solution of the forward problem needs to be stored in order complete the summation. This is potentially extremely memory consuming, but for the $2 \mathrm{D}$ problems considered in this work this has been possible. However, we remark that one could also write the forward solution to disc and then load it as necessary when solving the adjoint problem. Or instead of storing the all time steps of the forward solution, parts of the solution history can be recomputed while carying out the adjoint solution. This way, the solutions are only stored at the selected time steps which are called check-points. Interested readers are referred to the works of ${ }^{5152}$ regarding algorithms for check-point placement. Here it is noted that, while a check-pointing scheme is both memory and disc-space efficient, it comes at the cost of having to solve the forward problem twice.

The derivatives $\frac{\partial \mathbf{A}_{\mathbf{0}}}{\partial \overline{\mathrm{s}}}, \frac{\partial \mathbf{A}}{\partial \overline{\mathbf{s}}}$ and $\frac{\partial \mathbf{B}}{\partial \overline{\mathbf{s}}}$ contain the partial derivatives of the finite element matrices $(\mathbf{K}, \mathbf{M}$ and $\mathbf{C})$ with respect to the physical level set field $\overline{\mathbf{s}}$. For simplicity, these partial derivatives are approximated by a finite difference method using a central difference scheme. We remark that, the computational cost of carrying out rigorous analytic differentiation of these partial derivatives exceeds the computational cost of the perturbations involved in the finite difference method. For an in-depth discussion of the differentiation of cut element matrices, the reader is referred to 33153 . It is also noted that the sensitivity calculation in equation 61 is only done in the cut elements, since the terms $\frac{\partial \mathbf{A}_{0}}{\partial \overline{\mathbf{s}}}, \frac{\partial \mathbf{A}}{\partial \overline{\mathbf{s}}}$ and $\frac{\partial \mathbf{B}}{\partial \overline{\mathbf{s}}}$ are zero elsewhere. For completeness, the matrices $\frac{\partial \mathbf{A}_{\mathbf{0}}}{\partial \overline{\mathbf{s}}}, \frac{\partial \mathbf{A}}{\partial \overline{\mathbf{s}}}$ and $\frac{\partial \mathbf{B}}{\partial \overline{\mathbf{s}}}$ are written here as

$$
\begin{aligned}
& \frac{\partial \mathbf{A}}{\partial \overline{\mathbf{s}}}=\left[\begin{array}{ccc}
{\left[\frac{\partial \mathbf{K}}{\partial \bar{s}}+a_{6} \frac{\partial \mathbf{M}}{\partial \overline{\mathbf{s}}}+a_{3} \frac{\partial \mathbf{C}}{\partial \bar{s}}\right]} & \\
0 & 0 \\
0 & & 0
\end{array}\right], \quad \frac{\partial \mathbf{A}_{0}}{\partial \overline{\mathbf{s}}}=\left[\begin{array}{ccc}
0 & & \\
& 0 & \\
\frac{\partial \mathbf{K}}{\partial \overline{\mathbf{s}}} & \frac{\partial \mathbf{C}}{\partial \overline{\mathbf{s}}} & \frac{\partial \mathbf{M}}{\partial \overline{\mathbf{s}}}
\end{array}\right] \\
& \frac{\partial \mathbf{B}}{\partial \overline{\mathbf{s}}}=\left[\begin{array}{ccc}
{\left[-a_{6} \frac{\partial \mathbf{M}}{\partial \overline{\mathbf{s}}}-a_{3} \frac{\partial \mathbf{C}}{\partial \overline{\mathbf{s}}}\right]\left[-a_{4} \frac{\partial \mathbf{M}}{\partial \bar{s}}+a_{1} \frac{\partial \mathbf{C}}{\partial \overline{\mathbf{s}}}\right]\left[-a_{5} \frac{\partial \mathbf{M}}{\partial \overline{\mathbf{s}}}+a_{2} \frac{\partial \mathbf{C}}{\partial \overline{\mathbf{s}}}\right]} \\
0 & 0 & 0 \\
0 & 0 & 0
\end{array}\right]
\end{aligned}
$$

After calculating the sensitivities $\frac{\mathrm{d} \Phi}{\mathrm{d} \overline{\mathrm{s}}}$, the gradient with respect to the design variable $\mathbf{s}$ is obtained from the following chain

$$
\frac{\mathrm{d} \Phi}{\mathrm{d} \mathbf{s}}=\frac{\mathrm{d} \Phi}{\mathrm{d} \overline{\mathbf{s}}} \frac{\partial \overline{\mathbf{s}}}{\partial \overline{\mathbf{s}}_{\mathbf{c}}} \frac{\partial \overline{\mathbf{s}}_{\mathbf{c}}}{\partial \tilde{\mathbf{s}}_{\mathbf{c}}} \frac{\partial \tilde{\mathbf{s}}_{\mathbf{c}}}{\partial \tilde{\mathbf{s}}} \frac{\partial \tilde{\mathbf{s}}}{\partial \mathbf{s}}
$$

Above partial derivatives describe the operations involved in linking the physical level set $\overline{\mathbf{s}}$ to the design variable $\mathbf{s}$ in which they are applied in reverse order to obtain the gradient of the objective with respect to the design variable $\frac{\mathrm{d} \Phi}{\mathrm{ds}}$. Here the partial derivatives $\frac{\partial \overline{\mathbf{s}}}{\partial \overline{\mathbf{s}}_{\mathbf{c}}}$ and $\frac{\partial \tilde{s}_{\mathbf{c}}}{\partial \widetilde{\mathbf{s}}}$ are easily calculated since they describe interpolating the variables from element centers to nodes and from nodes to element centers, respectively. The term $\frac{\partial \overline{\mathbf{s}}_{\mathbf{c}}}{\partial \tilde{\mathbf{s}}_{\mathbf{c}}}$ deals with the utilized PDE filter and its implementation is thoroughly

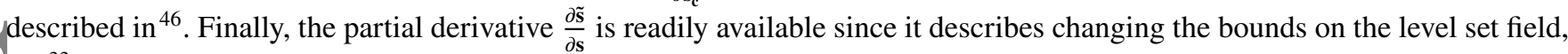
c.f. 33 .

\section{6 | NUMERICAL EXAMPLES}

The numerical investigations are conducted on the DTU Sophia cluster made up of 555 compute nodes each equipped with two AMD EPYC 735116 core CPUS and 128GB RAM. A maximum of two nodes, i.e. 64 cores, are used since the MPI parallelized direct solver MUMPS $\left({ }^{44 / 45}\right)$ is used for the solution to the state problem because direct solution methods are inherently sequential and cannot scale indefinitely. For all the presented optimization examples the code was allowed to run for 1500 design iterations which took no more than 5 hours to complete. We remark that the convergence of the objective function for all examples was monotone with small and insignificant oscillations towards the end. The settings for the MMA algorithm is for all examples: 
an MMA asymptote initialization of 0.5, an MMA asymptote increase of 1.2, an MMA asymptote decrease of 0.7, an MMA constraint penalization parameter of $c=1000$ and an outer move limit of $0.5 \%$.

\section{1 | Gradient validation}

This fist numerical example concerns a validation study for the implemented discrete adjoint approach. Furthermore, in order to motivate the discrete adjoint approach for transient optimization, the section also compares the method against the sensitivities that are obtained with the commonly used semi-discrete approach ${ }^{15}$. In the semi-discrete approach derivation for the adjoint equation and the subsequent gradient calculation is done analytically in time, i.e. before the temporal discretization, using an already spatially discretized system of equations. This means the utilized time integration scheme does not play a role in the derivation of the adjoint equation, i.e. the residual form of the chosen time stepping scheme is not considered. The derived adjoint equation therefore only has the same form as the spatially distretized forward problem (Eq. 23). This means that one is free to use a different time stepping algorithm for the adjoint problem, although the common practise is to reuse the one chosen for the forward problem.

This section considers a straightforward problem setup to validate and compare the calculated sensitivities in which the objective function is simply the summation of the absolute downstream acoustic pressure in the objective region over all time

steps given as

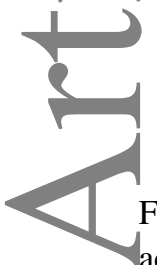

$$
\Phi=\sum_{n=0}^{N}\left[\int_{\Omega_{o b j}}\left|p^{n}\right| \mathrm{d} \Omega\right] \Delta t
$$

Figure 6 shows a schematic illustration of the design problem. The left most boundary is excited with an incoming sinusoidal acoustic plane wave with an amplitude of $p_{i n}=1 \mathrm{kPa}$. The top and the bottom boundaries are set as hard-walls while the right most boundary realizes an open boundary. The location where the objective function is evaluated is highlighted in the illustration with blue. A simple circular shaped structure is placed in the middle of the domain for which the calculated sensitivities at its interface will be investigated.

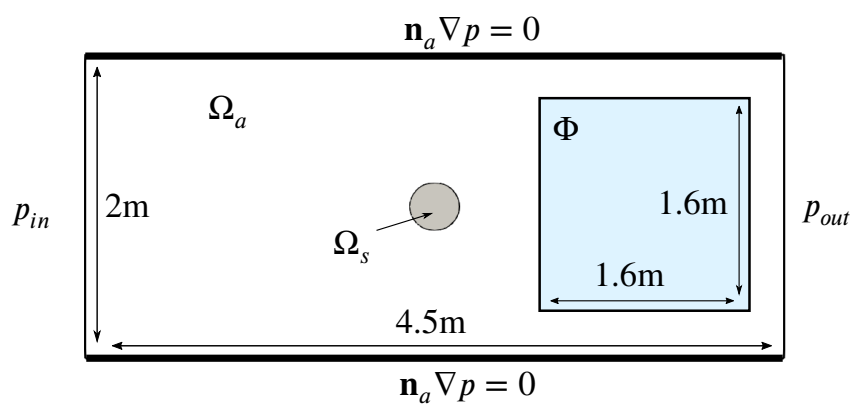

FIGURE 6 Schematic illustration of the sensitivity validation case showing the boundary conditions of the problem. Gray color shows the initial circle structure having the center at $(2.25 \mathrm{~m}, 1.0 \mathrm{~m})$ with a radius of $0.16 \mathrm{~m}$. Blue region illustrates the region where the objective function is evaluated.

Table 1 lists the material properties for the structural and acoustic domains, respectively. Regular quad elements having the edge length of $0.02 \mathrm{~m}$ is utilized for the computational mesh and the time step size is set to $\Delta t=0.05 \mathrm{~s}$ for the calculations.

\begin{tabular}{ccccc}
\hline$E[\mathrm{~Pa}]$ & $v$ & $\rho_{s}\left[\mathrm{~kg} / \mathrm{m}^{3}\right]$ & $c_{a}[\mathrm{~m} / \mathrm{s}]$ & $\rho_{a}\left[\mathrm{~kg} / \mathrm{m}^{3}\right]$ \\
\hline 1000 & 0.3 & 15 & 1.0 & 1.0 \\
\hline
\end{tabular}

TABLE 1 Material properties considered for the structural and acoustic media. 
For the first comparison, the calculated sensitivities using both methods are checked against a backward finite difference calculation. To this end, a random design variable contained in a cut element is chosen for investigation. The time step size $\Delta t$ is then held constant and a different number of time steps are utilized to illustrate the effect of the number of time steps on the quality of the calculated sensitivities.

Figure 7 presents the result of the finite difference check comparison for semi and fully discrete adjoint methods. As it can be seen from the figure, the finite difference check for the discrete adjoint method demonstrates the correct first order convergence as the step size for finite difference is decreased. This result was expected since the discrete sensitivity analysis always produces consistent sensitivities where the quality of the gradients does not depend on the utilized time step size or the number of time steps. The correct convergence behavior for the sensitivities calculated using the semi discrete approach on the other hand approached the first order convergence with an increasing the number of time steps.

In addition to finite difference comparison, the sensitivities that are calculated using both methods are numerically compared to each other. To this end, the calculated sensitivities for the design variables located on the lower half of the circular structure are extracted and compared to each other.
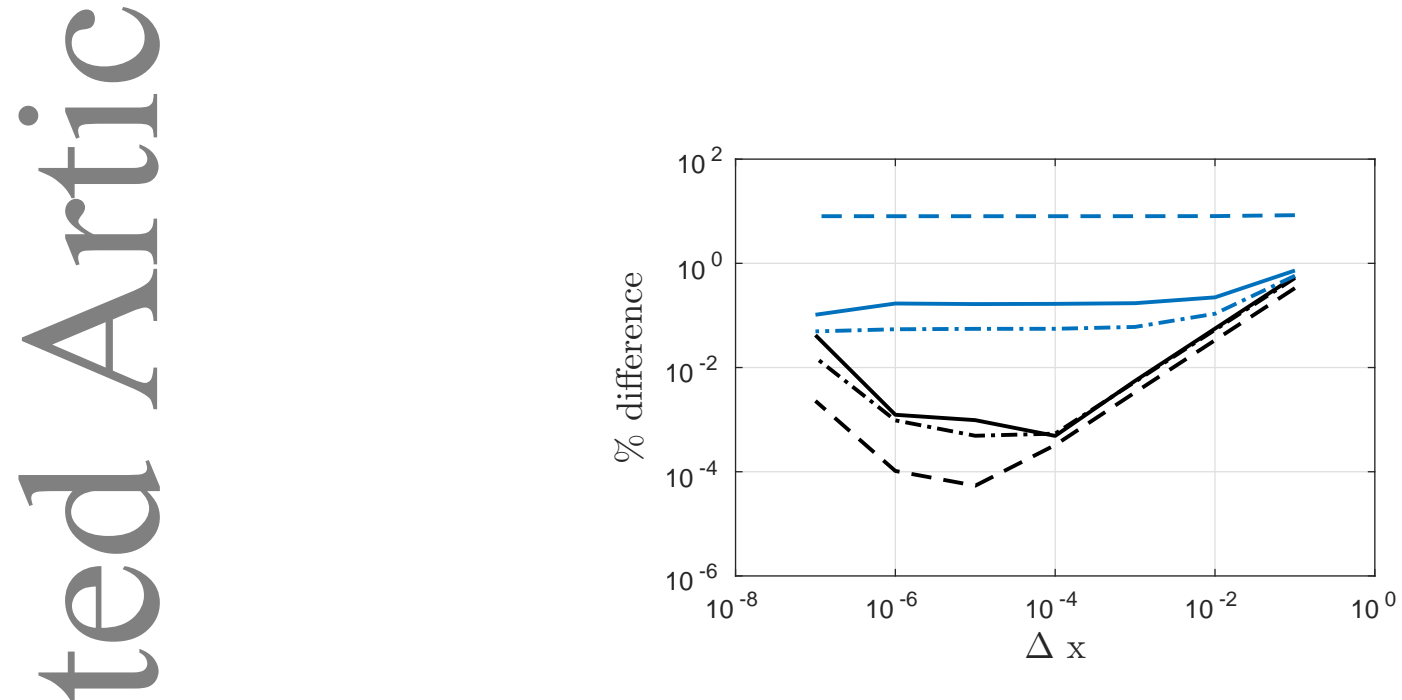

FIGURE 7 Finite difference check for validating the calculated sensitivities. Blue color specifies the semi-discrete adjoint melthod and the black color is the discrete adjoint method. For both methods, dashed lines are 50 time steps, solid lines are 100 time steps and dash-dot lines are 200 time steps.

( )

The calculation considers 50 time steps for both methods and the result is presented in the Figure 8 . It can be seen from the figure that sensitivities follow the same trend in magnitude. However, the difference becomes more critical at the locations where the calculated gradients change sign. It is seen that the inconsistent gradients produced with semi-discrete approach result in wrong signed sensitivities which would drive the design update in optimization towards the opposite direction. These findings motivate the use of the fully discrete adjoint approach for calculating consistent gradients.

\section{2 | Benchmark problem comparison}

The second example concerns the solution to a benchmark design problem taken from the seminal paper on vibroacoustic topology optimization ${ }^{25}$. The problem deals with the design of an elastic partitioner placed in an acoustic duct under a steady state assumption. The loading is through an incoming plane wave from the left and the duct is terminated with an open boundary to the right, c.f. Figure 9 The goal is to minimize the radiated acoustic pressure in the blue domain shown in the problem sketch. The amplitude of the incident wave is set to $p_{i n}=1 \mathrm{kPa}$ and a single frequency of $f=1 / \pi \mathrm{Hz}$ is used to generate the input signal. The material properties of both structure and acoustic medium can be seen in table 1 and the problem is solved for a total of $90 \mathrm{~s}$ using a time step of $\Delta t=0.006 \mathrm{~s}$, i.e. a total of 1500 time steps. The objective function can be written in terms of the 


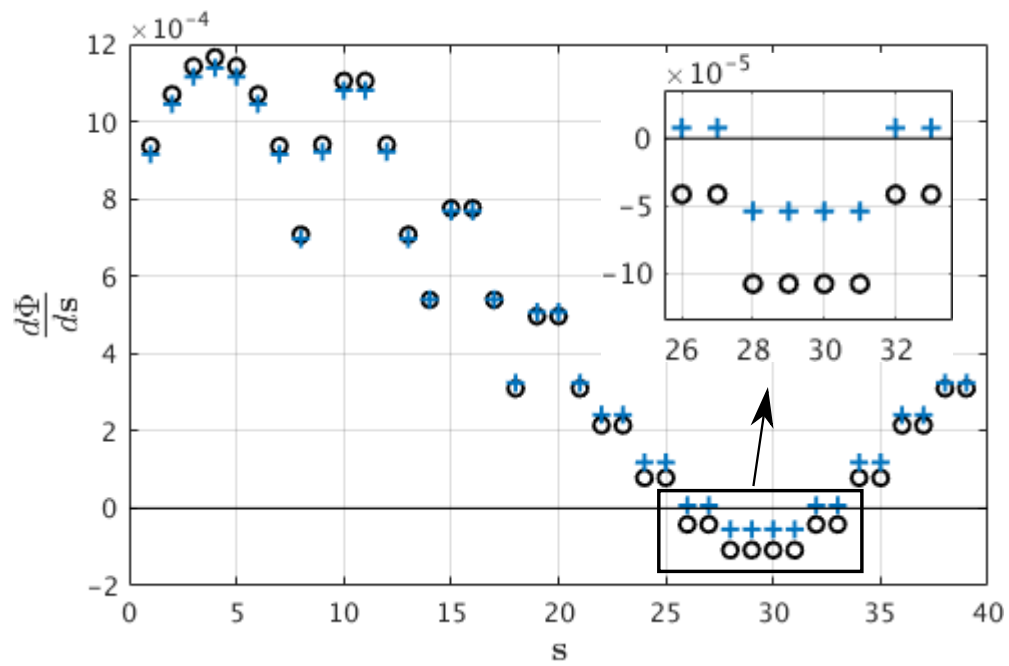

FIGURE 8 Calculated sensitivities $\frac{\mathrm{d} \Phi}{\mathrm{ds}}$ on the nodes of the mesh which are cut by the zero level set. Blue markers show the - semi-discrete sensitivity calculation and black markers illustrate the discrete sensitivity calculation.
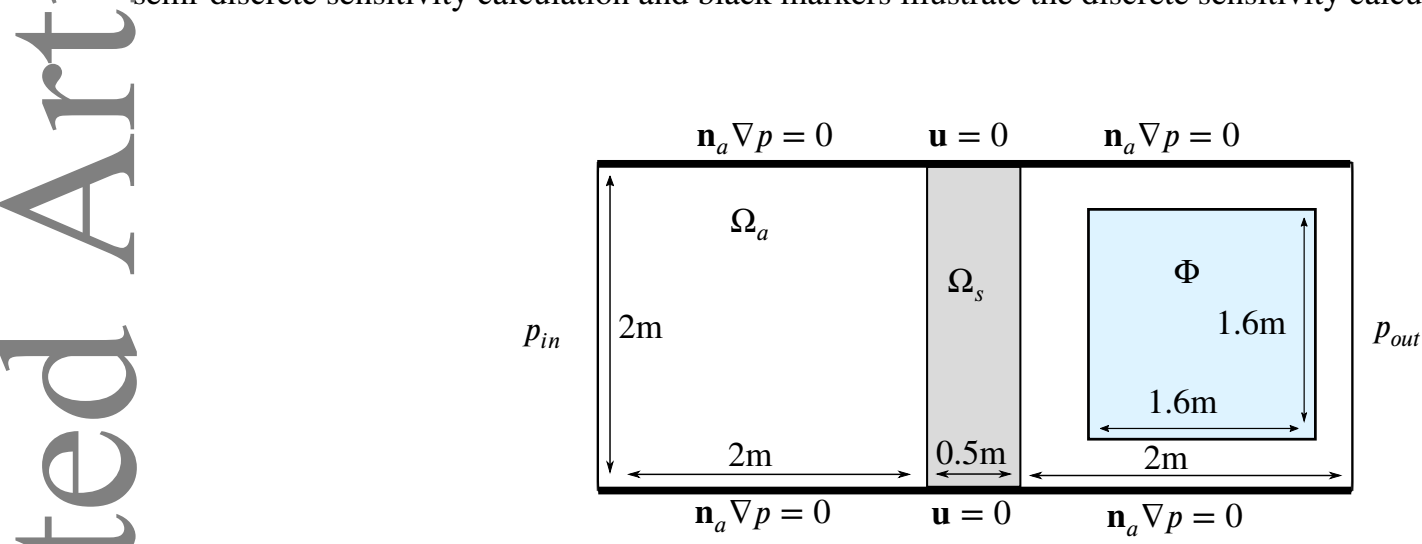

FIGURE 9 Schematic illustration of the benchmark example showing the boundary conditions of the optimization problem. Gray color shows the design domain and blue color illustrates the region where the objective function is evaluated. (1)

absolute acoustic pressure over the considered time span as

$$
\Phi=\sum_{n=n_{0}}^{N}\left[\int_{\Omega_{o b j}}\left|p^{n}\right| \mathrm{d} \Omega\right] \Delta t
$$

Note, that since the original benchmark problem is posed under a steady state assumption, the initial transient effects are omitted from this example by only summing over the final part of the time span. That is, the objective function calculation starts at the time step $n_{0}$ corresponding the time 70s. After this time the system response gets into an approximate steady state region, c.f. the interval between the two red lines in Fig. 10 Moreover, a volume constraint of $65 \%$ of the design domain is considered for the optimization. The computational domain is meshed with regular quad elements having the edge length of $0.02 \mathrm{~m}$ and the filter radius is set to $r=0.06 \mathrm{~m}$ throughout the optimization.

Figures 11a and $11 \mathrm{~b}$ present the initial guess supplied to the optimization and the optimized design, respectively. As it can be seen from the figure, the optimized design realizes approximately $53.4 \%$ performance increase compared to the initial guess where the previously reported "hour glass" shape ${ }^{25}$ is obtained.

Figure 10 compares the transient response of the volume averaged acoustic pressure over the objective calculation domain for the initial guess and the optimized design. The reported performance increase is also apparent from the figure in which the peaks of the transmitted acoustic pressure are approximately halved for the optimized design throughout the considered time span. 


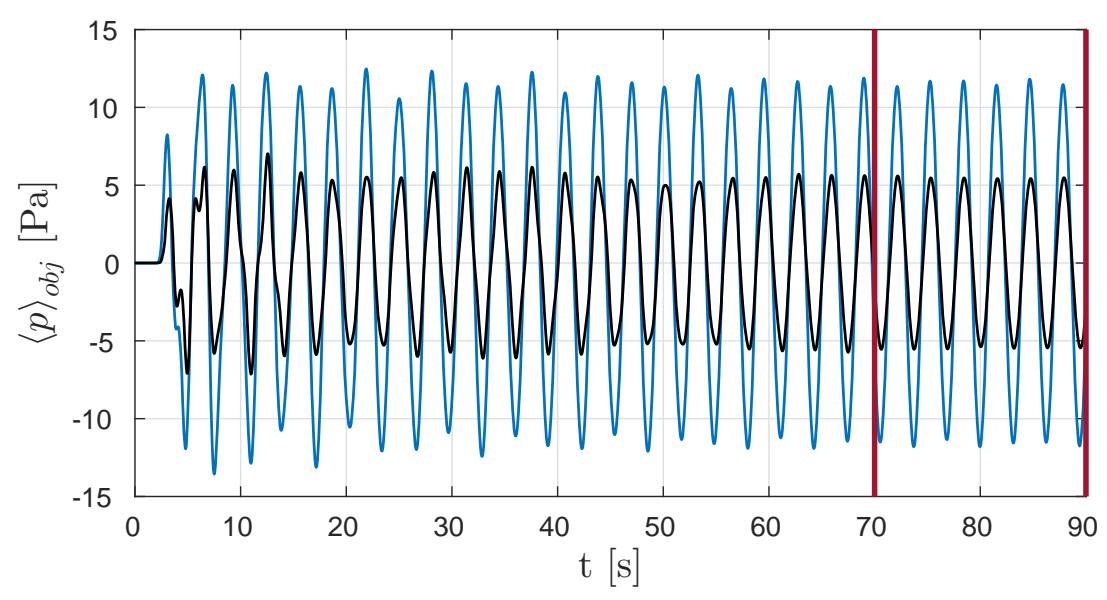

FIGURE 10 Averaged transmitted acoustic pressure calculated at the objective region. Blue lines is the initial design, black line is the optimized design. the vertical red lines illustrate the time span where the objective is calculated.

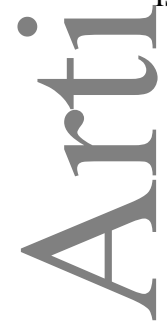

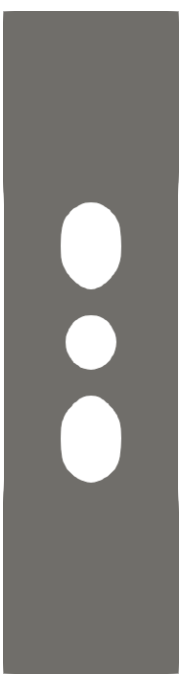

(a)

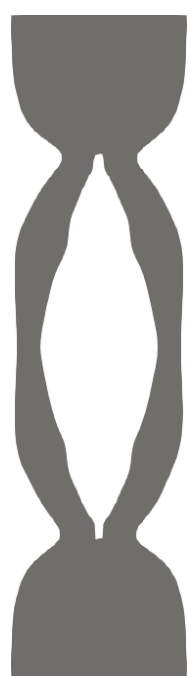

(b)

FIGURE 11 Benchmark example. (a) Initial design, $\Phi=627.41$ [Ns]. (b) optimized design, $\Phi=292.06$ [Ns].

Moreover, Figure 12 shows the transient acoustic pressure response (Figure 12a given in sound pressure level (SPL) and the corresponding scaled structural deformation (Figure $12 \mathrm{~b}$ ) at the last time step. Here, the SPL is calculated as

$$
\mathrm{SPL}=20 \log _{10}\left[\frac{|p(t)|}{2 \times 10^{-5}}\right] \quad[\mathrm{dB}]
$$

As it can be seen from Figure $12 \mathrm{~b}$ the structure is designed such that only the left-most bar is excited by the plane wave and hence the majority of the acoustic signal is reflected towards to inlet in order to minimize the transmitted downstream acoustic pressure. Here it is noted that, due to the nature of shape optimization, the developed optimization framework for coupled vibroacoustic problems exhibits strong sensitivity to the initial configuration. For the present case, the initial design given in Figure 11a was chosen as a result of experimenting with different initial hole configurations. All of the trials produced designs similar to the optimized structure given in Figure $11 \mathrm{~b}$ However, the given initial design (Figure 11a) resulted in the best performing elastic partitioner. 


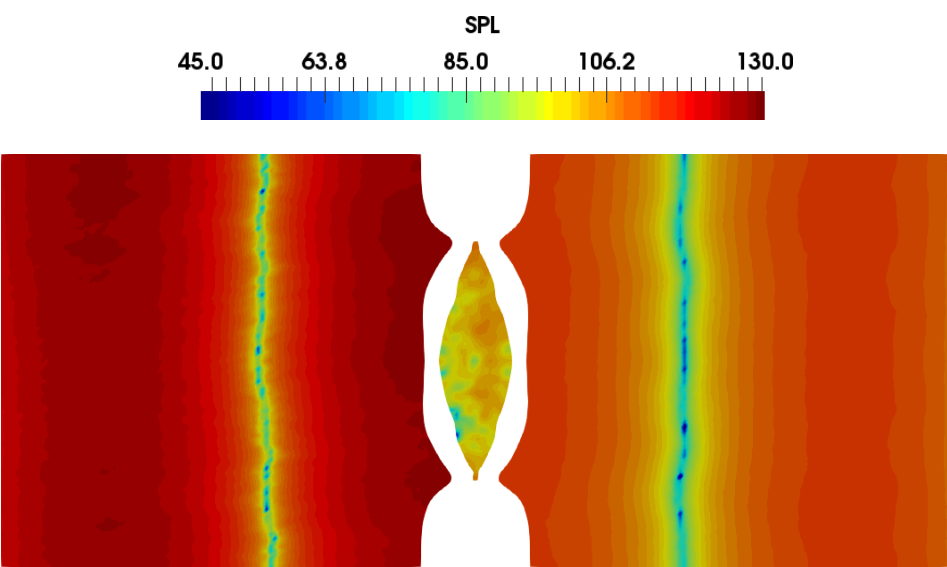

(a)

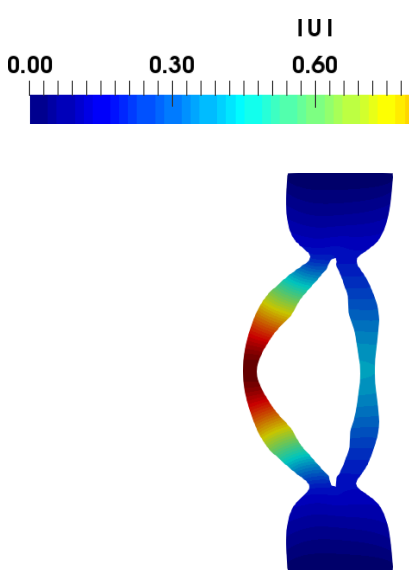

(b)

FIGURE 12 Transient acoustic pressure response and the corresponding structural deformation. (a) Sound pressure level

- SPL [dB]. (b) displacement magnitude $|\mathbf{u}|[\mathrm{m}]$.

\section{1 \\ 6.3 | Acoustic pulse shaping}

Having verified both sensitivities and the proposed transient optimization framework on a benchmark problem, the remainder of the paper is devoted to the design of vibroacoustic devices that utilizes the temporal problem formulation. To this end, a pulse shaping design problem inspired by the $1 \mathrm{D}$ counterpart from ${ }^{16}$ is employed and extended to 2D using both point and averaged line objectives.

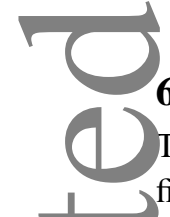

\subsection{1 | Pulse shaping at a point}

The first example considers the design of a structure where the transmitted acoustic pressure pulse at a specified point is to be fitted to a desired pulse shape after passing through a structural partitioner. Since the present case deals with the shape of the acoustic pulse rather than the specific pressure oscillations, the calculation of the pulse shape has been done through an envelope extraction of the recorded signal.

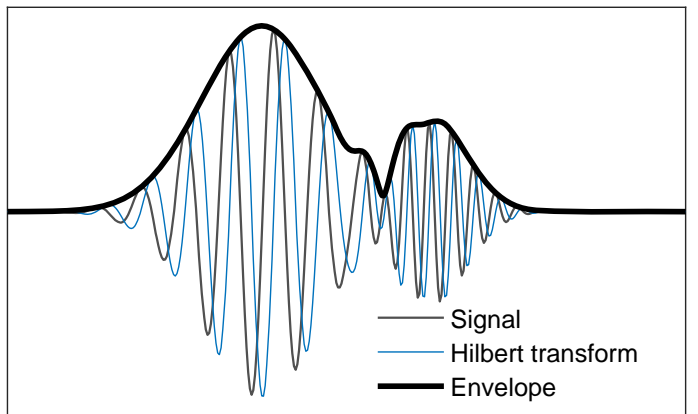

FIGURE 13 An example acoustic pulse showing the original signal, its Hilbert transform and the extracted envelope.

For a single frequency modulated signal, extracting the envelope information in topology optimization has been done in 54 . However, the current work utilizes the approach presented in $\frac{16}{16}$ in which the Hilbert transform is applied to transform and extract the envelope information. This way, the envelope of an any signal containing a broad range of frequencies can be calculated. Figure 13 illustrates an example signal where the Hilbert transform of the signal and the extracted envelope are shown. The 


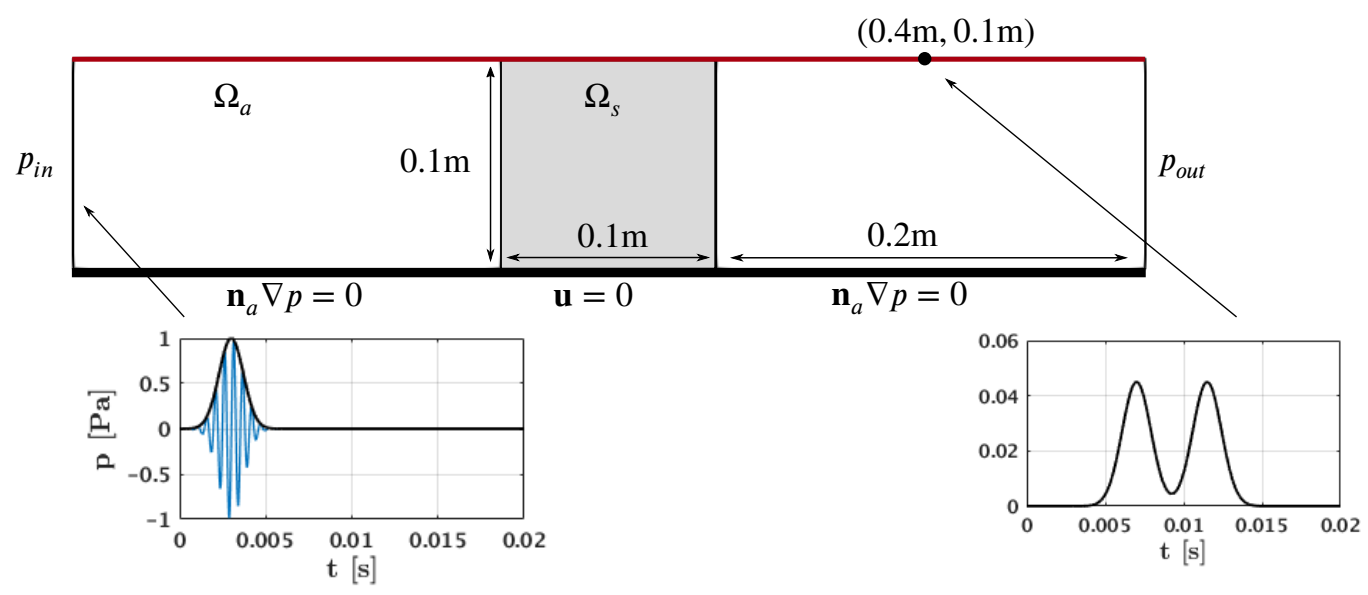

FIGURE 14 Schematic illustration of the optimization case for acoustic pulse shaping at a point showing the boundary conditions of the optimization problem. Top red line specifies the symmetry plane and gray color shows the design domain. Input acoustic pulse and the desired pulse shape at the specified point are also shown.

(נ)

envelope calculation is done as

$$
e(t)=\sqrt{p_{p}^{2}+\hat{p}_{p}^{2}}
$$

where $p_{p}$ is the recorded transmitted acoustic pressure at a given location (point) and the hat notation used in $\hat{p}_{p}$ specifies the Hilbert transform of the recorded acoustic pressure.

The schematic illustration in Figure 14 shows the computational domain along with the considered boundary conditions. As it can be seen from the figure, the bottom boundary is set as hard-wall condition for the acoustic pressure and the structure is considered to be clamped. The top boundary is set as a symmetry plane for both physics in order to reduce the computational cost. The left and right boundaries are absorbing boundaries in which the incoming acoustic pulse at the left most boundary is shown in the figure. The acoustic pulse is realized as a plane wave and is composed of frequencies roughly between $1000 \mathrm{~Hz}$ and $3000 \mathrm{~Hz}$. The desired pulse shape for the acoustic pressure at the specified location is also shown in the figure. The material used for the structural domain is rubber-like whereas the acoustic domain is taken as air, c.f. table2. In order to reflect the loss mechanism of the real world, and to stabilize the optimization process, a Rayleigh damping is employed as described in section 2.1. The Rayleigh parameters $\alpha_{d}$ and $\beta_{d}$ are calculated as 55

$$
\begin{aligned}
& \alpha_{d}=2 \zeta \frac{\omega_{1} \omega_{2}}{\omega_{1}+\omega_{2}} \\
& \beta_{d}=2 \zeta \frac{1}{\omega_{1}+\omega_{2}}
\end{aligned}
$$

where the damping ratio $\zeta$ is taken to be $\zeta=0.1$. Moreover, the two natural frequencies $\omega_{1}$ and $\omega_{2}$ are assumed to be $\omega_{1}=$ $16002 \pi \mathrm{rad} / \mathrm{s}$ and $\omega_{2}=22002 \pi \mathrm{rad} / \mathrm{s}$. For modeling and the subsequent optimization, the time step size is set to $\Delta t=2 \times 10^{-5} \mathrm{~s}$. The overall calculation is chosen as $0.02 \mathrm{~s}$ which results in 1000 time steps for both forward and adjoint solutions. The filter radius is set to $r=1 \times 10^{-3} \mathrm{~m}$ and the computational domain is meshed with regular quad elements having the edge length of $2 \times 10^{-3} \mathrm{~m}$.

\begin{tabular}{ccccc}
\hline$E[\mathrm{~Pa}]$ & $v$ & $\rho_{s}\left[\mathrm{~kg} / \mathrm{m}^{3}\right]$ & $c_{a}[\mathrm{~m} / \mathrm{s}]$ & $\rho_{a}\left[\mathrm{~kg} / \mathrm{m}^{3}\right]$ \\
\hline $50 \times 10^{6}$ & 0.4 & 1000 & 343 & 1.21 \\
\hline
\end{tabular}

TABLE 2 Material properties considered for the structural and acoustic domains. 
The objective function uses a least squares type formulation in order to minimize the difference between the calculated and the desired pulse shape as

$$
\Phi=\sum_{n=0}^{N}\left[\left(e^{n}\right)^{2}-\left(f^{n}\right)^{2}\right]^{2} \Delta t
$$

where $f(t)$ is the desired envelope shape. The above terms are squared in order to avoid division by zero when calculating the adjoint source $\frac{\partial \Phi}{\partial \mathbf{U}}$ which is due to $p(t)=0$ at the start of the calculations. Here the vector $\mathbf{U}$ in $\frac{\partial \Phi}{\partial \mathbf{U}}$ is given in the Eq. 43 which contains the solution vector $\mathbf{v}=[\mathbf{u}, \mathbf{p}]^{T}$ and its first and second time derivatives due to the Newmark algorithm. The derivation of adjoint source term $\frac{\partial \Phi}{\partial \mathbf{U}}$ is described in details in $\frac{16}{}$.

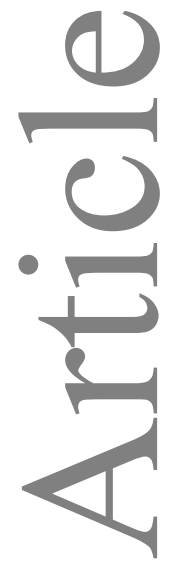

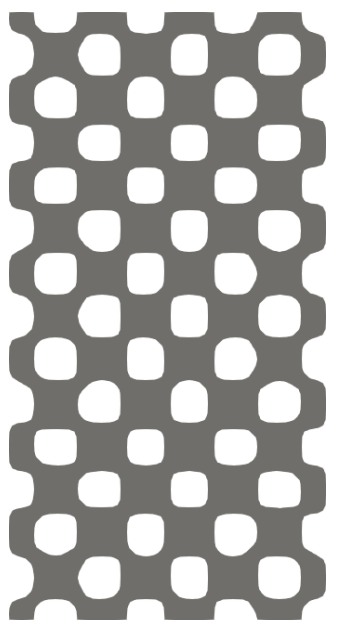

(a)

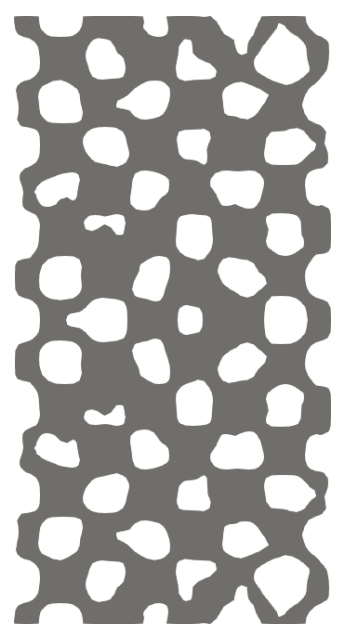

(c)

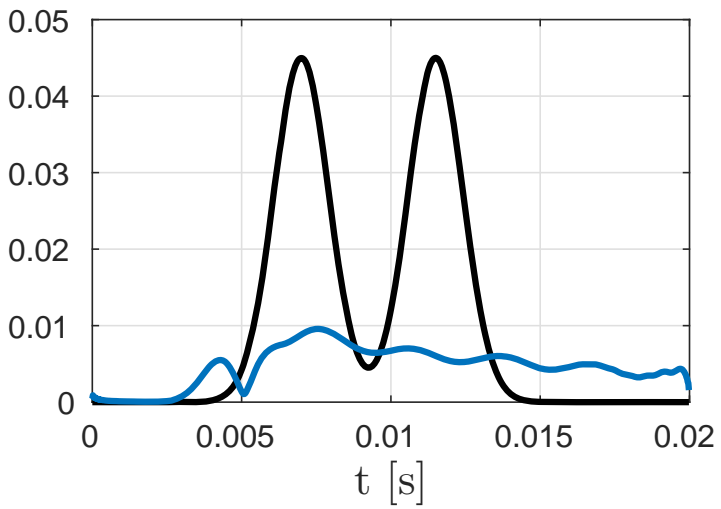

(b)

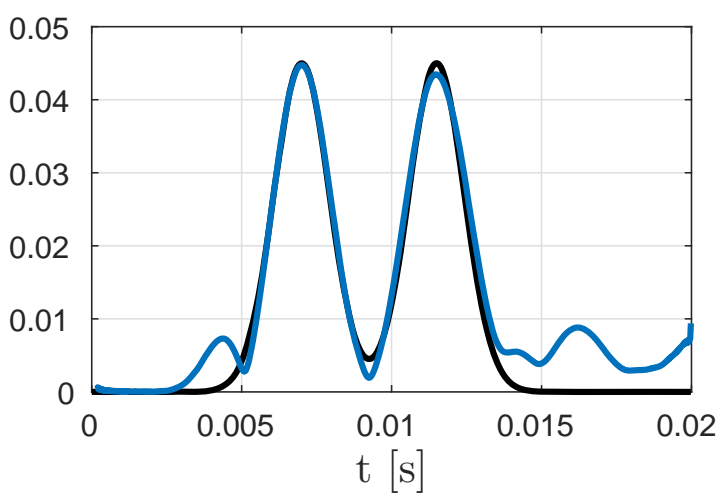

(d)

FIGURE 15 Optimization for acoustic pulse shaping at a point. (a) Initial configuration $\Phi^{*}=0.9258$. (b) Calculated acoustic pulse shape with the initial configuration, black line is the desired pulse shape and blue line is the calculated pulse shape. (c) Optimized design $\Phi^{*}=0.0044$. (d) Calculated acoustic pulse shape with the optimized design, black line is the desired pulse shape and blue line is the calculated pulse shape.

The reported objective values are given in a normalized form in order to better asses the performance of the designs as

$$
\Phi^{*}=\frac{\Phi}{\sum_{n=0}^{N}\left(f^{n}\right)^{4} \Delta t}
$$


Figures $15 \mathrm{a}$ and $15 \mathrm{c}$ shows the intial guess and the optimized designs, respectively. It can be seen from Figure $15 \mathrm{~b}$ that the incoming acoustic pulse is completely smeared out after passing through the initial design configuration. The transmitted signal can be seen to have an almost constant envelope shape, although slowly attenuating, after it first hits the measurement point. The poor performance is also evident from the objective measure which very low, i.e. $\Phi^{*}=0.9258$ :

The optimized design, on the other hand, realizes an objective of $\Phi^{*}=0.00448 \%$ and by visual inspection of Figure $15 \mathrm{~d}$, it is clear that the acoustic pulse shape follows closely the desired envelope shape. Comparing the initial and the optimized design configurations (Figures $15 \mathrm{a}$ and $15 \mathrm{c}$. respectively) shows that the optimization only changes the shape of the cavities present in the initial design which clearly illustrates a strong initial guess dependency of the level set based shape optimization method. The limited design change throughout the optimization and the resulting performance increase for the optimized design, also indicates that the coupled vibroacoustic performance in a point is quite sensitive to small design variations. This also points to the fact that tailoring the response in a point seems to be an easy optimization problem as well.
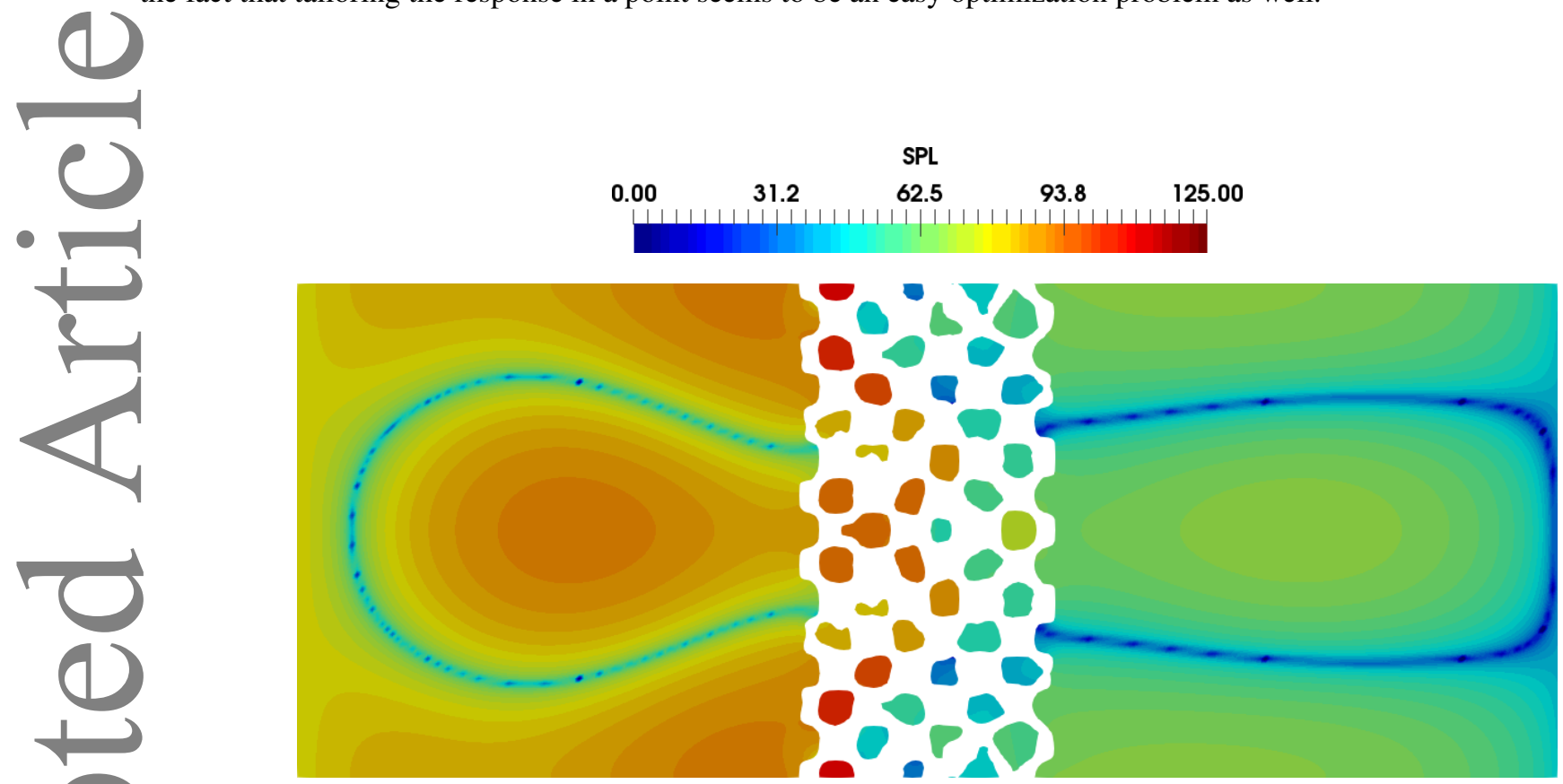

FIGURE 16 Sound pressure level $[\mathrm{dB}]$ contours of the optimized design for the first pulse shaping example. The given pressure field is from the time 0.007 [s].

In summary, the result in Figure 15 shows that the proposed optimization setup can successfully tailor the transient response of the coupled system in a point. Furthermore, inspecting a snapshot of the acoustic SPL at time 0.007s, as shown in Figure 16 reveals that the vibroacoustic interaction caused by the shape of the optimized design, focuses the transmitted acoustic wave towards to middle of the channel where the acoustic response is measured. Note that the selected snapshot corresponds to the time of the second peak of the desired envelope shape.

It should be noted that if the optimized design is analyzed using a stiffer material, the envelope fitting is completely lost and hence that the chosen structure indeed utilizes the vibroacoustic coupling to achieve the desired performance. Finally, it is important to stress that the optimized design is quite sensitive to boundary variations, or defects, and hence that a geometric robust approach should be included when considering fabrication. This could, as already mentioned, be done by e.g. the robust level set approach of ${ }^{33}$, but is deeemed outside the scope of this manuscript.

\subsection{2 | Pulse shaping over a line}

The last numerical example extends the pulse shaping problem from a point objective to an objective given as the average over a line. This is considered to be a harder optimization problem as the optimizer can no longer focus the acoustic energy at a point, but must match the target shape over the entire line in question. Hence, the envelope calculation has to be modified to 


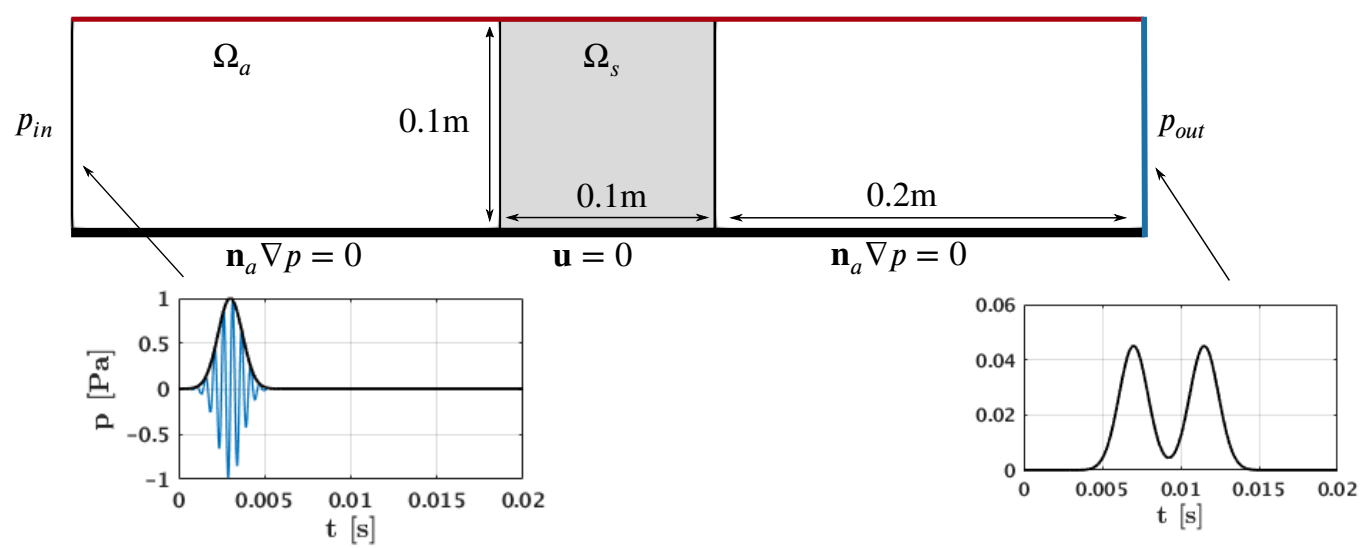

FIGURE 17 Schematic illustration of the optimization case for acoustic pulse shaping at the outlet showing the boundary conditions of the optimization problem. Top red line specifies the symmetry plane and gray color shows the design domain. Input acoustic pulse and the desired pulse shape at the outlet are also shown.

accommodate the new objective, i.e.

$$
e(t)=\sqrt{\langle p\rangle_{\text {out }}^{2}+\langle\hat{p}\rangle_{\text {out }}^{2}}
$$

where $\langle p\rangle_{\text {out }}$ is the acoustic pressure signal averaged over the outlet boundary for at each time step and $\langle\hat{p}\rangle_{\text {out }}$ is its Hilbert transformed counterpart. The averaging is simply calculated as

$$
\langle p\rangle_{\text {out }}=\frac{\int_{\Gamma_{\text {out }}} p(t) \mathrm{d} \Gamma}{\int_{\Gamma_{\text {out }}} \mathrm{d} \Gamma}
$$

The modified pulse shaping design problem is illustrated in Figure 17 in which the line used for the pressure averaging has been highlighted. The pulse excitation, number of time steps, total time, discretization, material and damping parameters, as well as the boundary conditions remain the same as those used in the previous design case.

This also goes for the objective function, however, in order to account for the pressure averaging, the adjoint source term is modified as

$$
\frac{\partial \Phi}{\partial \mathbf{U}}=\frac{\partial \Phi}{\partial\langle\mathbf{U}\rangle} \frac{\partial\langle\mathbf{U}\rangle}{\partial \mathbf{U}}
$$

where the first term on the right hand side concerns the derivative of the Hilbert transform operation wrt the averaged acoustic pressure and second the term, i.e. $\frac{\partial\langle\mathbf{U}\rangle}{\partial \mathbf{U}}$, deals with the averaging operation.

The initial configuration together with the optimized design can be seen in Figures $18 \mathrm{a}$ and $18 \mathrm{c}$ respectively. Inspecting the initial configuration and its corresponding envelope measured at the outlet boundary, c.f. Figure 18b, makes it clear that the signal is almost killed (in an average sense) when reaching the outlet after the initial pulse has passed. This is contrary to that observed for the point objective and is due to internal reflections in the initial structure resulting in highly non-uniform wave pattern reaching the outlet boundary. This is also evident in the initial objective value which is $\Phi^{*}=0.9945$. Again, the optimization process is capable of producing a design with a good fit to the desired pulse, c.f. Figure $18 \mathrm{~d}$ This is also clear from the objective value of the optimized design which reaches $\Phi^{*}=0.0045$. More surprisingly is the topological change observed in the optimized design compared to the initial configuration. That is, the initial configuration has a total of 45 holes including those that touch the top and bottom boundary. On the other hand, the optimized has a total of 47 holes and has in general undergone a more noticeable topological change compared to that of the point objective problem. As no hole generation scheme is included in the presented work, the new holes are a consequence of the existing holes having merged at an early point in the process to form large holes. The boundaries of these larger holes then subsequently meet at multiple points which leads to the topological change. It is also worth to mention that the central half-circular holes at the top and bottom boundaries have moved away from the interface and thus also resulted in a topology change. Finally, it also seen that two very small holes remain in the optimized design. However, by inspecting the SPL field plot in Figure 19 it is seen that the intensity in these holes are negligible and hence, they have no significant influence on the performance of the pulse shaping device. 


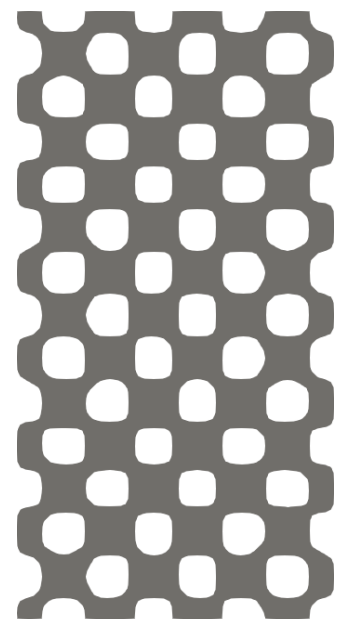

(a)

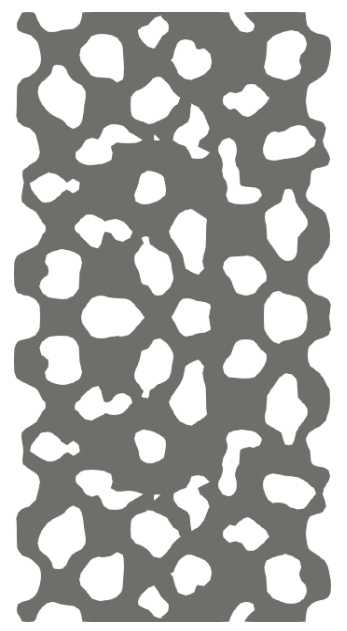

(c)

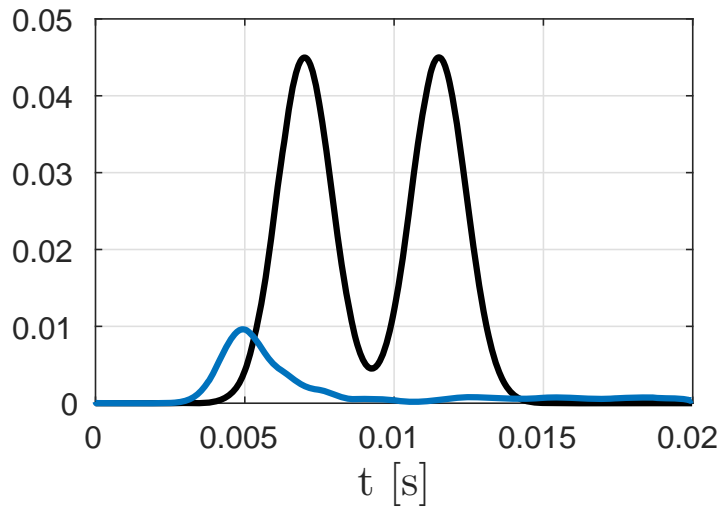

(b)

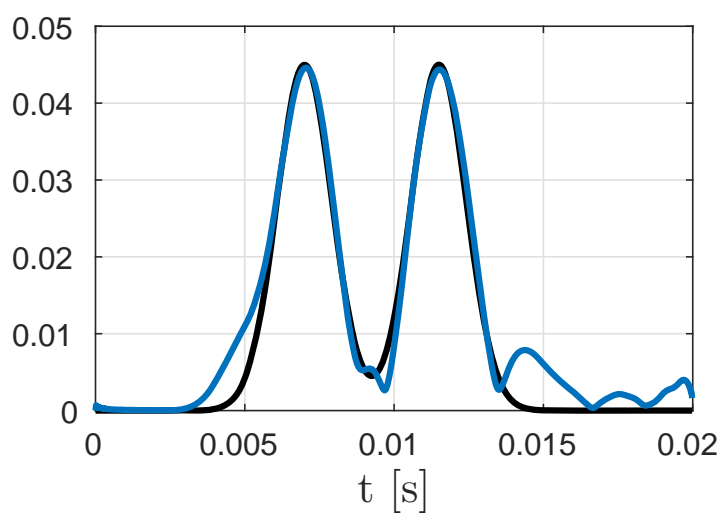

(d)

FIGURE 18 Optimization for acoustic pulse shaping at the outlet. (a) Initial configuration $\Phi^{*}=0.9945$. (b) Calculated acoustic pulse shape with the initial configuration, black line is the desired pulse shape and blue line is the calculated pulse shape. (c) Optimized design $\Phi^{*}=0.0045$. (d) Calculated acoustic pulse shape with the optimized design, black line is the desired pulse shape and blue line is the calculated pulse shape.

A couple of common observations are valid for both point and line objective formulations of the pulse shaping problem. First, it is clear from the results that neither of the designs are capable of delaying the pulse impact on measurement point/line. That is, from the transmission graphs in Figures $15 \mathrm{~b}, 15 \mathrm{~d}$ [18b and $18 \mathrm{~d}$ it is seen that the initial impact of the acoustic pressure occurs at the same time for both initial and optimized configurations. Based on the findings of ${ }^{16}$ it is expected that a significantly wider design domain capable of representing multiple repeated Bragg gratings would be able to delay the impact of the pulse. However, this would require a much larger numerical model for which more efficient solution strategies have to be employed for an efficient solution. Secondly, it is also clear that the used objective measure favours to match the target envelope at the peaks and not at the side lobes. This can again be related to the design domain size in the sense that multiple reflecting surfaces would be necessary to efficiently reflect the input signal such that the transmitted signal would die out after desired target envelope time has passed. 


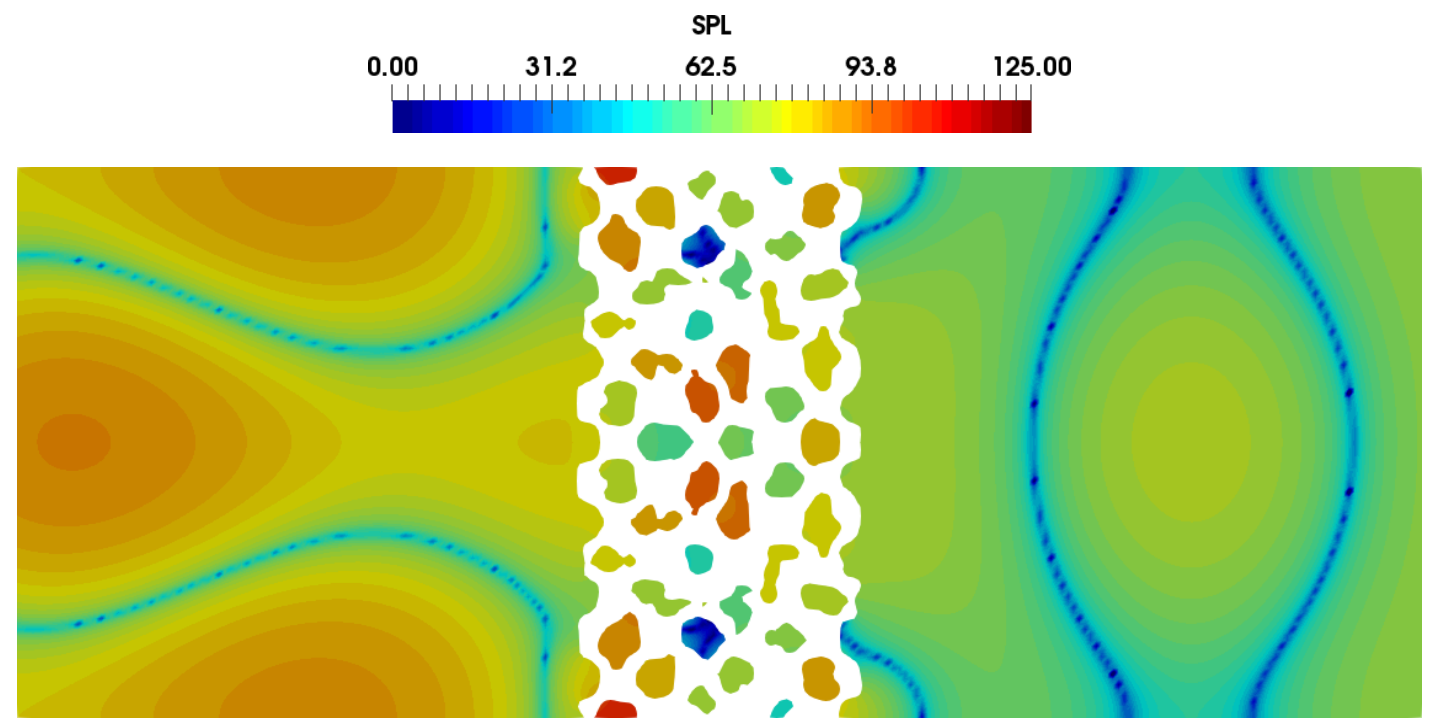

FIGURE 19 Sound pressure level [dB] contours of the optimized design for the second pulse shaping example. The given pressure field is from the time 0.007 [s].

\section{7 | CONCLUSIONS}

The article presents generalized shape optimization for transient vibroacoustic problems. The work utilizes an explicit level set approach where the nodal level set values are directly linked to the mathematical design variables to allow for the use of efficient nonlinear programming tools e.g. the (MMA) algorithm. Instead of following the classical level set approach in which the remeshing is commonly done to capture the design for carrying out the analysis, the current work employs an immersed boundary cut element method which operates on a fixed background mesh. Using this method, complex geometries can be accurately modelled for strongly coupled physics such as acoustic mechanical interaction problems. To accurately model the coupling interface between the two physics, the cut element method uses a special integration scheme without adding extra degrees of freedom to the system. Therefore the method can be included into existing parallel FEM frameworks with minimal effort on the developnt, while maintaining a good load balancing throughout the optimization process. For the optimization, the discrete adjoint method is utilized for calculating the gradients of the objective and constraint functions. Overall the derivation of the discrete adjoint method has been done considering the utilized time integration scheme which is the Newmark algorithm. However, the approach can readily be applied for any other time integration scheme since the derivation is given in discrete residual form. The considered numerical examples starts with the validation of the calculated sensitivities within the discrete adjoint approach. The example compares the calculated sensitivities considering a test problem where the discrete and commonly used semi-discrete methods are compared. It is illustrated that the quality of the calculated gradients using the semi-discrete approach depends on the number of time steps used for the forward model and the adjoint equation. It is shown for an extreme case, the semi-discrete approach can lead to wrong signed sensitivities because of inconsistencies in this approach. The fully discrete approach, on the other hand, always produces consistent sensitivities. The developed transient framework is then employed on a benchmark problem which deals with designing of an elastic partitioner in order to reduce the transmitted acoustic pressure. For timeharmonic topology optimization of vibroacoustic problems, the problem has been introduced in ${ }^{25}$. By using a transient inlet sinusoidal acoustic plane wave and optimizing after the initial transient effects have passed, the previously reported "hour-glass" shaped structure is obtained. A transient application concerning the design of an acoustic pulse shaping device is considered to demonstrate the tailoring of an acoustic reading at a specified point. The structure is subjected to an incoming acoustic wave packet consisting of frequencies between $1000 \mathrm{~Hz}$ and $3000 \mathrm{~Hz}$. The envelope of the transmitted acoustic pressure at the specified point is then calculated and fitted to a predefined shape. Compared to the initial configuration, the optimization realizes a 99.5\% performance increase. The same problem setup is then modified to the tailoring of an envelope shape of the transmitted acoustic pressure averaged over a line. The optimized design shows a similar performance increase and has a final objective close to zero. More interestingly, the design obtained using the line objective also reveals significant topological changes. That is, the initial configuration has 45 holes whereas the optimized shape has 47 . This is possible due to the versatility of the used 
level approach, which, to a large extend is based on ideas from density based topology optimization. Finally, the presented work will open the way for the optimization of complex transient signals containing broad frequency content as well as broadband filter applications for vibroacoustic problems.

\section{ACKNOWLEDGEMENTS}

The authors would like to express their gratitude to the Villum Foundation and the InnoTop VILLUM investigator project for access to the computing resources. The authors have no competing interests.

\section{REFERENCES}

1. Bendsøe MP, Sigmund O. Topology Optimization - Theory, Methods, and Applications. Springer Verlag . 2003.

2. Svanberg K. The method of moving asymptotes-a new method for structural optimization. International Journal for Numerical Methods in Engineering 1987; 24(2): 359-373. doi: 10.1002/nme.1620240207, 10.1002/(ISSN)1097-0207

3. Lundgaard C, Alexandersen J, Zhou M, Andreasen CS, Sigmund O. Revisiting density-based topology optimization for fluid-structure-interaction problems. Struct. Multidiscip. Optim. 2018; 58(3): 969-995. doi: 10.1007/s00158-018-1940-4

4. Bourdin B. Filters in topology optimization. International Journal for Numerical Methods in Engineering 2001; 50(9): 2143-2158. doi: $10.1002 / n m e .116$

5. Bruns TE, Tortorelli DA. Topology optimization of non-linear elastic structures and compliant mechanisms. Computer Methods in Applied Mechanics and Engineering 2001; 190(26-27): 3443-3459. doi: 10.1016/s0045-7825(00)00278-4

6. Guest JK, Prévost JH, Belytschko T. Achieving minimum length scale in topology optimization using nodal design variables and projection functions. International Journal for Numerical Methods in Engineering 2004; 61(2): 238-254. doi: $10.1002 / \mathrm{nme} .1064$

7. Wang F, Lazarov BS, Sigmund O. On projection methods, convergence and robust formulations in topology optimization. Structural and Multidisciplinary Optimization 2011; 43(6): 767-784. doi: 10.1007/s00158-010-0602-y

Aage N, Andreassen E, Lazarov BS, Sigmund O. Giga-voxel computational morphogenesis for structural design. Nature 2017; 550(7674): 84-86. doi: 10.1038/nature23911

9. Kontoleontos EA, Papoutsis-Kiachagias EM, Zymaris AS, Papadimitriou DI, Giannakoglou KC. Adjoint-based constrained topology optimization for viscous flows, including heat transfer. Engineering Optimization 2013; 45(8): 941-961. doi: 10.1080/0305215X.2012.717074

10. Dilgen CB, Dilgen SB, Fuhrman DR, Sigmund O, Lazarov BS. Topology optimization of turbulent flows. Computer Methods in Applied Mechanics and Engineering 2018; 331: 363-393. doi: 10.1016/j.cma.2017.11.029

11. Dühring MB, Jensen JS, Sigmund O. Acoustic design by topology optimization. Journal of Sound and Vibration 2008; 317(3-5): 557-575. doi: 10.1016/j.jsv.2008.03.042

12. Park J, Wang S. Noise reduction for compressors by modes control using topology optimization of eigenvalue. Journal of Sound and Vibration 2008; 315(4-5): 836-848. doi: 10.1016/j.jsv.2008.01.064

13. Christiansen RE, Sigmund O. Experimental validation of systematically designed acoustic hyperbolic meta material slab exhibiting negative refraction. Applied Physics Letters 2016; 109(10): 101905. doi: 10.1063/1.4962441

14. Søndergaard MB, Pedersen CB. Applied topology optimization of vibro-acoustic hearing instrument models. J. Sound Vib. 2014; 333(3): 683-692. doi: 10.1016/j.jsv.2013.09.029

15. Dahl J, Jensen JS, Sigmund O. Topology optimization for transient wave propagation problems in one dimension. Structural and Multidisciplinary Optimization 2008; 36(6): 585-595. doi: 10.1007/s00158-007-0192-5 
16. Lazarov BS, Matzen R, Elesin Y. Topology optimization of pulse shaping filters using the Hilbert transform envelope extraction. Structural and Multidisciplinary Optimization 2011; 44(3): 409-419. doi: 10.1007/s00158-011-0642-y

17. Pollini N, Lavan O, Amir O. Adjoint sensitivity analysis and optimization of hysteretic dynamic systems with nonlinear viscous dampers. Struct. Multidiscip. Optim. 2018; 57(6): 2273-2289. doi: 10.1007/s00158-017-1858-2

18. Jensen JS. Space-time topology optimization for one-dimensional wave propagation. Comput. Methods Appl. Mech. Eng. 2009; 198(5-8): 705-715. doi: 10.1016/j.cma.2008.10.008

19. Wang W, Munro D, Wang CCL, Keulen vF, Wu J. Space-time topology optimization for additive manufacturing. Struct. Multidiscip. Optim. 2020; 61(1): 1-18. doi: 10.1007/s00158-019-02420-6

20. Jensen JS, Sigmund O. Topology optimization for nano-photonics. Laser and Photonics Reviews 2011; 5(2): 308-321. doi: 10.1002/lpor.201000014

21. Sigmund O. Design of multiphysics actuators using topology optimization - Part I. Computer Methods in Applied Mechanics and Engineering 2001; 190(49-50): 6577-6604. doi: 10.1016/s0045-7825(01)00251-1

22. Alexandersen J, Sigmund O, Aage N. Large scale three-dimensional topology optimisation of heat sinks cooled by natural convection. International Journal of Heat and Mass Transfer 2016; 100: 876-891. doi: 10.1016/j.ijheatmasstransfer.2016.05.013

23. Dilgen SB, Dilgen CB, Fuhrman DR, Sigmund O, Lazarov BS. Density based topology optimization of turbulent flow heat transfer systems. Structural and Multidisciplinary Optimization 2018; 57(5): 1905-1918. doi: 10.1007/s00158-018-1967-6

24. Lawry M, Maute K. Level set topology optimization of problems with sliding contact interfaces. Struct. Multidiscip. Optim. 2015; 52: 1107-1119. doi: 10.1007/s00158-015-1229-9

25. Yoon GH, Jensen JS, Sigmund O. Topology optimization of acoustic-structure interaction problems using a mixed finite element formulation. International Journal for Numerical Methods in Engineering 2007; 70(9): 1049-1075. doi: $10.1002 / \mathrm{nme} .1900$

26. Osher S, Sethian JA. Fronts propagating with curvature-dependent speed: Algorithms based on Hamilton-Jacobi formulations. J. Comput. Phys. 1988; 79(1): 12-49. doi: 10.1016/0021-9991(88)90002-2

27. Sethian JA, Wiegmann A. Structural Boundary Design via Level Set and Immersed Interface Methods. Journal of Computational Physics 2000; 163(2): 489-528. doi: 10.1006/jcph.2000.6581

28. Noguchi Y, Yamamoto T, Yamada T, Izui K, Nishiwaki S. A level set-based topology optimization method for simultaneous design of elastic structure and coupled acoustic cavity using a two-phase material model. Journal of Sound and Vibration 2017; 404: 15-30. doi: 10.1016/j.jsv.2017.05.040

29. Isakari H, Kuriyama K, Harada S, Yamada T, Takahashi T, Matsumoto T. A topology optimisation for three-dimensional acoustics with the level set method and the fast multipole boundary element method. Mechanical Engineering Journal 2014; 1(4): CM0039-CM0039. doi: 10.1299/mej.2014cm0039

30. Shu L, Yu Wang M, Ma Z. Level set based topology optimization of vibrating structures for coupled acoustic-structural dynamics. Computers and Structures 2014; 132: 34-42. doi: 10.1016/j.compstruc.2013.10.019

31. Desai J, Faure A, Michailidis G, Parry G, Estevez R. Topology optimization in acoustics and elasto-acoustics via a level-set method. Journal of Sound and Vibration 2018; 420: 73-103. doi: 10.1016/j.jsv.2018.01.032

32. Dilgen CB, Dilgen SB, Aage N, Jensen JS. Topology optimization of acoustic mechanical interaction problems: a comparative review. Structural and Multidisciplinary Optimization 2019; 60(2): 779-801. doi: 10.1007/s00158-019-02236-4

33. Andreasen CS, Elingaard MO, Aage N. Level set shape and topology optimization by density methods using cut elements with length scale control. Structural and Multidisciplinary Optimization, Accepted 2020. 
34. Düster A, Parvizian J, Yang Z, Rank E. The finite cell method for three-dimensional problems of solid mechanics. Computer Methods in Applied Mechanics and Engineering 2008; 197(45): 3768-3782. doi: 10.1016/j.cma.2008.02.036

35. Daux C, Moës N, Dolbow J, Sukumar N, Belytschko T. Arbitrary branched and intersecting cracks with the extended finite element method. International Journal for Numerical Methods in Engineering 2000; 48(12): 1741-1760. doi: 10.1002/1097-0207(20000830)48:12<1741::AID-NME956>3.0.CO;2-L

36. Belytschko T, Parimi C, Moës N, Sukumar N, Usui S. Structured extended finite element methods for solids defined by implicit surfaces. International Journal for Numerical Methods in Engineering 2003; 56(4): 609-635. doi: 10.1002/nme.686

37. Düster A, Parvizian J, Yang Z, Rank E. The finite cell method for three-dimensional problems of solid mechanics. Computer Methods in Applied Mechanics and Engineering 2008; 197(45-48): 3768-3782. doi: 10.1016/j.cma.2008.02.036

38. Zienkiewicz O, Taylor R. The finite element method. Butterworth Heinemann, . 2000.

39. Newmark NM. A method of computation for structural dynamics. Journal of the engineering mechanics division 1959; 85(3): 67-94.

40. Jacob BP, Ebecken NFF. An optimized implementation of the Newmark/Newton-Raphson algorithm for the time integration of non-linear problems. Communications in Numerical Methods in Engineering 1994; 10(12): 983-992. doi: $10.1002 / \mathrm{cnm} .1640101204$

41. Balay S, Abhyankar S, Adams MF, et al. PETSc Web page. http://www.mcs.anl.gov/petsc, 2018.

42. Balay S, Abhyankar S, Adams MF, et al. PETSc Users Manual. Tech. Rep. ANL-95/11 - Revision 3.10, Argonne National Laboratory; 2018.

43. Balay S, Gropp WD, McInnes LC, Smith BF. Efficient Management of Parallelism in Object Oriented Numerical Software Libraries. In: Arge E, Bruaset AM, Langtangen HP., eds. Modern Software Tools in Scientific ComputingBirkhäuser Press; 1997: 163-202.

44. Amestoy PR, Duff IS, Koster J, L'Excellent JY. A Fully Asynchronous Multifrontal Solver Using Distributed Dynamic Scheduling. SIAM Journal on Matrix Analysis and Applications 2001; 23(1): 15-41.

45. Amestoy PR, Guermouche A, L'Excellent JY, Pralet S. Hybrid scheduling for the parallel solution of linear systems. Parallel Computing 2006; 32(2): 136-156.

46. Lazarov BS, Sigmund O. Filters in topology optimization based on Helmholtz-type differential equations. International Journal for Numerical Methods in Engineering 2011; 86(6): 765-781. doi: 10.1002/nme.3072

47. Lorensen WE, Cline HE. Marching cubes: A high resolution 3D surface construction algorithm. Computer Graphics (acm) 1987; 21(4): 163-169.

48. www.comsol.com . COMSOL Multiphysics Reference Manual, version 5.5. 2020.

49. Aage N, Lazarov BS. Parallel framework for topology optimization using the method of moving asymptotes. Structural and Multidisciplinary Optimization 2013; 47(4): 493-505. doi: 10.1007/s00158-012-0869-2

50. Aage N, Andreassen E, Lazarov BS. Topology optimization using PETSc: An easy-to-use, fully parallel, open source topology optimization framework. Struct. Multidiscip. Optim. 2015; 51(3): 565-572. doi: 10.1007/s00158-014-1157-0

51. Griewank A, Walther A. Algorithm 799: Revolve: An implementation of checkpointing for the reverse or adjoint mode of computational differentiation. Acm Transactions on Mathematical Software 2000; 26(1): 19-45.

52. Wang Q, Moin P, Iaccarino G. Minimal repetition dynamic checkpointing algorithm for unsteady adjoint calculation. Siam Journal on Scientific Computing 2009; 31(4): 2549-2567. doi: 10.1137/080727890

53. Sharma A, Villanueva H, Maute K. On shape sensitivities with heaviside-enriched XFEM. Structural and Multidisciplinary Optimization 2017; 55(2): 385-408. doi: 10.1007/s00158-016-1640-x 
54. Yang L, Lavrinenko A, Hvam JM, Sigmund O. Design of one-dimensional optical pulse-shaping filters by time-domain topology optimization. Applied Physics Letters 2009; 95(26): 261101. doi: 10.1063/1.3278595

55. Puthanpurayil AM, Lavan O, Carr AJ, Dhakal RP. Elemental damping formulation: an alternative modelling of inherent damping in nonlinear dynamic analysis. Bulletin of Earthquake Engineering 2016; 14(8): 2405-2434. doi: 10.1007/s10518016-9904-9 